\title{
Matching with Myopic and Farsighted Players
}

Citation for published version (APA):

Herings, P. J-J., Mauleon, A., \& Vannetelbosch, V. (2017). Matching with Myopic and Farsighted Players. Maastricht University, Graduate School of Business and Economics. GSBE Research Memoranda No. $011 \mathrm{https}: / /$ doi.org/10.26481/umagsb.2017011

Document status and date:

Published: 01/05/2017

DOI:

10.26481/umagsb.2017011

Document Version:

Publisher's PDF, also known as Version of record

\section{Please check the document version of this publication:}

- A submitted manuscript is the version of the article upon submission and before peer-review. There can be important differences between the submitted version and the official published version of record.

People interested in the research are advised to contact the author for the final version of the publication, or visit the DOI to the publisher's website.

- The final author version and the galley proof are versions of the publication after peer review.

- The final published version features the final layout of the paper including the volume, issue and page numbers.

Link to publication

\footnotetext{
General rights rights.

- You may freely distribute the URL identifying the publication in the public portal. please follow below link for the End User Agreement:

www.umlib.nl/taverne-license

Take down policy

If you believe that this document breaches copyright please contact us at:

repository@maastrichtuniversity.nl

providing details and we will investigate your claim.
}

Copyright and moral rights for the publications made accessible in the public portal are retained by the authors and/or other copyright owners and it is a condition of accessing publications that users recognise and abide by the legal requirements associated with these

- Users may download and print one copy of any publication from the public portal for the purpose of private study or research.

- You may not further distribute the material or use it for any profit-making activity or commercial gain

If the publication is distributed under the terms of Article $25 \mathrm{fa}$ of the Dutch Copyright Act, indicated by the "Taverne" license above, 


\section{Maastricht University}

P. Jean-Jacques Herings,

Ana Mauleon,

Vincent Vannetelbosch

Matching with Myopic and Farsighted Players

$\mathrm{RM} / 17 / 011$

\section{GSBE}

Maastricht University School of Business and Economics

Graduate School of Business and Economics

P.O Box 616

NL- 6200 MD Maastricht

The Netherlands 


\title{
Matching with Myopic and Farsighted Players
}

\author{
P. Jean-Jacques Herings* Ana Mauleon ${ }^{\dagger}$ \\ Vincent Vannetelbosch ${ }^{\ddagger}$
}

April 19, 2017

\begin{abstract}
We study stable sets for marriage problems under the assumption that players can be both myopic and farsighted. We introduce the new notion of the myopic-farsighted stable set, which is based on the notion of a myopicfarsighted improving path. A myopic-farsighted stable set is the set of matchings such that there is no myopic-farsighted improving path from any matching in the set to another matching in the set (internal stability) and there is a myopic-farsighted improving path from any matching outside the set to some matching in the set (external stability). For the special cases where all players are myopic and where all players are farsighted, our concept predicts the set of matchings in the core. When all men are myopic and the top choice of each man is a farsighted woman, we show that the singleton consisting of the woman-optimal stable matching is a myopic-farsighted stable set. The same result holds when all women are farsighted. We present examples where this is the unique myopic-farsighted stable set as well as examples of myopic-farsighted stable sets consisting of a core element different from the woman-optimal matching or even of a non-core element.
\end{abstract}

Key words: Marriage problems, stable sets, myopic and farsighted players. JEL classification: C70, C78.

*Department of Economics, Maastricht University, Maastricht, The Netherlands. E-mail: P.Herings@maastrichtuniversity.nl

${ }^{\dagger}$ CEREC, Saint-Louis University - Brussels; CORE, University of Louvain, Louvain-la-Neuve, Belgium. E-mail: ana.mauleon@usaintlouis.be

${ }^{\ddagger}$ CORE, University of Louvain, Louvain-la-Neuve; CEREC, Saint-Louis University - Brussels, Belgium. E-mail: vincent.vannetelbosch@uclouvain.be 


\section{Introduction}

Experimental and empirical studies in matching markets suggest that agents are heterogeneous with respect to their degree of farsightedness and confirm the underlying hypothesis that being unsophisticated is correlated with belonging to a disadvantaged group. ${ }^{1}$ Despite this evidence, the extant theoretical literature on matching markets has only proposed stability concepts assuming that players are homogeneous regarding their level of myopia or farsightedness.

The current paper proposes a solution concept for marriage problems that allows for the interaction between myopic and farsighted players. This allows us to study whether farsighted players are able to achieve a better outcome than myopic players. Our objective is to link the theoretical results regarding the stability of twosided matching markets with the experimental and empirical evidence regarding the question whether markets systematically favor a stable matching with particular characteristics.

A matching is stable if no individual player prefers to destroy an existing match and no pair of players prefers to form a match between them. Existing solution concepts for matching markets assume players to have the same degree of farsightedness and are not able to discriminate between different stable matchings. For matching markets populated by heterogeneous players, we demonstrate that farsighted players are able to achieve their preferred stable matching.

Experimentally, a number of papers analyze decentralized markets. Echenique and Yariv (2012) find that subjects are strategically sophisticated and show the impact of the cardinal representation of ordinal preferences on which stable match gets selected. Kagel and Roth (2000) analyze the transition from decentralized matching to centralized clearinghouses, when market features lead to inefficient matching through unraveling. Nalbantian and Schotter (1995) analyze several procedures for matching with transferable utilities, decentralized matching among them, where

\footnotetext{
${ }^{1}$ Basteck and Mantovani (2016) test subjects' cognitive ability and compare their allocation to schools under the Boston and the Deferred Acceptance mechanisms. They show subjects of lower cognitive ability are systematically harmed under Boston and that substantial ability segregation may result, with the top school enrolling up to 45 percent more high ability students than the worst school. These results confirm the underlying hypothesis that being unsophisticated is correlated with belonging to an already disadvantaged group, so that the Boston mechanism would selectively discriminate the weakest students.
} 
agents have private information about payoffs. Boudreau (2011) runs simulation experiments and shows that there are cases in which one side of the market has an inherent advantage over the other side in that their more-favored equilibrium is more likely to prevail when matching evolves in a decentralized manner. ${ }^{2}$

Following the cooperative game theory model of matching markets, ${ }^{3}$ the set of stable matchings coincides with the core in marriage problems. Gale and Shapley (1962) have shown that the core of a marriage problem is non-empty. Ehlers (2007) has characterized the von Neumann-Morgenstern (vNM) stable sets in marriage problems and has shown that the set of matchings in the core is a subset of any vNM stable set and a vNM stable set can contain matchings outside the core. Wako (2010) shows that the vNM stable set exists and is unique.

The standard dominance relation used to define vNM stable sets violates the assumption of coalitional sovereignty $(\mathrm{C}),{ }^{4}$ the property that an objecting coalition cannot enforce matches between members outside the coalition. A further criticism of the standard definition of the vNM stable set is that it does not take into account that a deviation by a coalition can be followed by further deviations. Herings, Mauleon, and Vannetelbosch (2017) follow the approach by van Deemen (1991) and Page and Wooders (2009) and define the stable set with respect to path dominance $(\mathrm{P})$, resulting in the pairwise $\mathrm{CP}$ vNM set. They show that in marriage problems there is a unique pairwise $\mathrm{CP}$ vNM set and that it coincides with the core.

The notions of core and vNM stable set are myopic notions since the players do not anticipate that individual and coalitional deviations are countered by subsequent deviations. These concepts are based on the direct dominance relation and neglect the destabilizing effect of indirect dominance relations as introduced by Harsanyi (1974) and Chwe (1994). Indirect dominance captures the idea that coalitions of farsighted players can anticipate the actions of other coalitions and consider the end matching that their deviations may lead to.

\footnotetext{
${ }^{2}$ There is a growing experimental literature studying centralized matching systems, e.g., Harrison and McCabe (1996), Chen and Sönmez (2006), Haruvy and Ünver (2007), Pais and Pintér (2008), Featherstone and Mayefsky (2011), Featherstone and Niederle (2011), and Echenique, Wilson and Yariv (2016).

${ }^{3}$ We refer to Roth and Sotomayor (1990) for a comprehensive overview on two-sided matching problems.

${ }^{4}$ Ray and Vohra (2015) express the same criticism towards the vNM stable set for nontransferable utility games.
} 
Based on the concept of indirect dominance, several solution concepts assume farsighted behavior of the players in matching models. Diamantoudi and Xue (2003) have shown that in hedonic games with strict preferences core partitions are always contained in the largest consistent set due to Chwe (1994). ${ }^{5}$ However, the largest consistent set may contain more matchings than those matchings that are in the core. Mauleon, Vannetelbosch, and Vergote (2011) characterize the vNM farsightedly stable sets as all singletons that contain a core element and show that the farsighted core, defined by Diamantoudi and Xue (2003) as the set of matchings that are not indirectly dominated by other matchings, can be empty. ${ }^{6}$

The extant theoretical literature is silent about the selection of stable matchings in case many such matchings exist. We argue here that one possible reason for this fact is the absence of a solution concept that allows for heterogeneity in the degree of myopia or farsightedness among players.

In the present paper, we propose the notion of myopic-farsighted stable set to study the matchings that are stable when myopic and farsighted players interact with each other. The new notion of myopic-farsighted stable set is based on the notion of a myopic-farsighted improving path. A myopic-farsighted improving path is a sequence of matchings that can emerge when farsighted players form or destroy links based on the improvement the end matching offers relative to the current matching while myopic players form or destroy links based on the improvement the next matching offers relative to the current matching. Each matching in the sequence differs from the previous one in that either a new match is formed or an existing match is destroyed.

A myopic-farsighted stable set is the set of matchings satisfying internal and external stability with respect to the notion of a myopic-farsighted improving path. That is, there is no myopic-farsighted improving path from any matching in the set to another matching in the set (internal stability) and there is a myopic-farsighted improving path from any matching outside the set to some matching in the set (external stability).

When all players are myopic, the myopic-farsighted stable set is equivalent to

\footnotetext{
${ }^{5}$ Other approaches to farsightedness in coalition and network formation are suggested by the work of Xue (1998), Mauleon and Vannetelbosch (2004), Page, Wooders, and Kamat (2005), Herings, Mauleon and Vannetelbosch (2004, 2009), and Page and Wooders (2009) among others.

${ }^{6}$ The farsighted core only exists when the core contains a unique matching and no other matching indirectly dominates the matching in the core.
} 
the pairwise CP vNM set of Herings, Mauleon, and Vannetelbosch (2017) and the unique myopic-farsighted stable set is equal to the core of the marriage problem. When all players are farsighted, the myopic-farsighted stable set is closely related to the vNM farsightedly stable set of Mauleon, Vannetelbosch, and Vergote (2011). The myopic-farsighted stable sets with only farsighted players are characterized as the singletons consisting of a core element.

We then turn to cases where the two sides of the market are heterogeneous in their degree of farsightedness. We fully analyze the typical example where the preferences of men and women are diametrically opposed and show that in case all players on one side are myopic and at least one player on the other side is farsighted, the optimal stable matching of the farsighted side constitutes the unique myopicfarsighted stable set. In all other cases, any core outcome and no other outcome is sustained by the myopic-farsighted stable set.

We assume next that the men are all myopic and the top choice of each man is a farsighted woman or to remain single. We also study the case where all women are farsighted without further assumptions on preferences. ${ }^{7}$ We show that under both sets of assumptions, the woman-optimal stable matching constitutes a myopicfarsighted stable set. The result implies that the presence of some farsighted women is enough to guarantee that the woman-optimal stable matching can always be reached, starting from any other matching, by means of a myopic-farsighted improving path. Thus, also the myopic women benefit from the presence of farsighted women.

Several papers in the matching literature (see for instance Diamantoudi and Xue (2003), Ehlers (2007), Mauleon, Vannetelbosch, and Vergote (2011), and Herings, Mauleon, and Vannetelbosch (2017) among others) point towards the core as the set of reasonable outcomes, but are not able to discriminate between different core elements. However, in these papers, no heterogeneity regarding the degree of farsightedness of players in the two sides of the market was considered. Both sides are assumed to be either myopic or farsighted. By assuming that one side of the market

\footnotetext{
${ }^{7}$ Using data on user attributes and interactions from an online dating site, Hitsch, Hortaçsu and Ariely (2010) estimate mate preferences and use the Gale-Shapley algorithm to predict stable matches. They show that the average difference between the users' first choice and the rank achieved by the Gale-Shapley algorithm is larger for men than for women. Hence, women equilibrium matches are closer to their first choice, compared with men. We therefore find it natural to think of the women as being more farsighted than the men.
} 
is more farsighted than the other side, we find that selection among core elements is possible.

However, we present examples to show that other myopic-farsighted stable sets can co-exist. We present an example where the man-optimal stable matching is different from the woman-optimal stable matching and show that also the set containing the man-optimal stable matching is a myopic-farsighted stable set. More surprisingly, we also provide an example showing that a set consisting of a single element not belonging to the core can be a myopic-farsighted stable set. This non-core element consists of a proper subset of the matches that are present in the womanoptimal stable matching and matches the farsighted women with the same partner as in the woman-optimal stable matching.

The paper is organized as follows. Section 2 introduces marriage problems and standard notions of stability. Section 3 defines the myopic-farsighted stable set and characterizes the implications of all possible constellations regarding farsightedness for the case where preferences of men and women are diametrically opposed. Section 4 studies societies where either all players are myopic or all players are farsighted as special cases. Section 5 establishes the main result that the woman-optimal stable matching is always a myopic-farsighted stable set when all men are myopic and have a farsighted woman or remaining single as their top choice and Section 6 presents the same result when all women are farsighted. Section 7 discusses the robustness of our main results. Section 8 concludes.

\section{Marriage Problems}

A marriage problem consists of a finite set of players $N$, partitioned into a set of men $M$ and a set of women $W$. The set of non-empty subsets of $N$ is denoted by $\mathcal{N}$. Each player $i \in N$ has a complete and transitive preference ordering $\succ_{i}$ over the players of opposite sex and the prospect of being alone. Preferences are assumed to be strict. Let $\succ=\left(\left(\succ_{m}\right)_{m \in M},\left(\succ_{w}\right)_{w \in W}\right)$ be a preference profile. We write $m \succ_{w} m^{\prime}$ if woman $w$ strictly prefers $m$ to $m^{\prime}, m \sim_{w} m^{\prime}$ if $w$ is indifferent between $m$ and $m^{\prime}$, and $m \succeq_{w} m^{\prime}$ if $m \succ_{w} m^{\prime}$ or $m \sim_{w} m^{\prime}$. Since preferences are assumed to be strict, $m \sim_{w} m^{\prime}$ implies $m=m^{\prime}$. Similarly, we write $w \succ_{m} w^{\prime}, w \sim_{m} w^{\prime}$, and $w \succeq_{m} w^{\prime}$. A marriage problem is a triple $(M, W, \succ)$.

A matching is a function $\mu: N \rightarrow N$ satisfying the following properties: 
(i) For every $m \in M, \mu(m) \in W \cup\{m\}$.

(ii) For every $w \in W, \mu(w) \in M \cup\{w\}$.

(iii) For every $i \in N, \mu(\mu(i))=i$.

The set of all matchings is denoted by $\mathcal{M}$. Given a matching $\mu \in \mathcal{M}$, player $i$ is said to be single if $\mu(i)=i$. A matching $\mu$ is individually rational if each player is acceptable to his or her partner, so for every $i \in N$ it holds that $\mu(i) \succeq_{i} i$. A matching $\mu$ that is not individually rational can be blocked by a player with an unacceptable partner. For a given matching $\mu$, a pair $\{m, w\}$ is said to form a blocking pair if $m$ and $w$ are not matched to one another but prefer one another to their partners at $\mu$, i.e. $w \succ_{m} \mu(m)$ and $m \succ_{w} \mu(w)$. A matching $\mu$ is stable if it is not blocked by any single player or any pair of players.

Given a matching $\mu \in \mathcal{M}$ with man $m \in M$ matched to woman $w \in W$, so $\mu(m)=w$, the matching $\mu^{\prime}$ that is identical to $\mu$, except that the match between $m$ and $w$ has been destroyed by either $m$ or $w$, is denoted by $\mu-(m, w)$. Given a matching $\mu \in \mathcal{M}$ such that $m \in M$ and $w \in W$ are not matched to each other, the matching $\mu^{\prime}$ that is identical to $\mu$, except that $(m, w)$ are now matched at $\mu^{\prime}$ and their partners at $\mu$, i.e., $\mu(w)$ and $\mu(m)$, are now singletons at $\mu^{\prime}$, is denoted by $\mu+(m, w)$.

For every $i \in N$, we extend the preference ordering $\succ_{i}$ over the player's potential partners to the set of matchings $\mathcal{M}$ in the following way. We say that player $i$ prefers the matching $\mu^{\prime}$ to the matching $\mu$ if $\mu^{\prime}(i) \succ_{i} \mu(i)$ and we write $\mu^{\prime} \succ_{i} \mu$. For $S \in \mathcal{N}$, $\mu(S)=\{\mu(i) \mid i \in S\}$ denotes the set of partners of players in $S$ at $\mu$. A coalition $S \in \mathcal{N}$ is said to block a matching $\mu \in \mathcal{M}$ if there exists a matching $\mu^{\prime} \in \mathcal{M}$ such that $\mu^{\prime}(S)=S$ and $\mu^{\prime} \succ_{S} \mu$, where $\mu^{\prime} \succ_{S} \mu$ is defined as $\mu^{\prime}(i) \succ_{i} \mu(i)$ for every $i \in S$. The core of the marriage problem $(M, W, \succ)$ consists of all matchings that are not blocked by any coalition. We denote the set of matchings that belong to the core by $C$.

It has been shown by Gale and Shapley (1962) that the core of a marriage problem is non-empty. Also, a matching is stable if and only if it is not blocked by a coalition of size one or two if and only if it belongs to the core, see Theorem 3.3 in Roth and Sotomayor (1990). Knuth (1976) has shown that the core of a marriage problem is a distributive lattice. In particular, there is a man-optimal stable matching $\mu^{\mathrm{M}}$ and a woman-optimal stable matching $\mu^{\mathrm{W}}$. For any matching $\mu$ in the core, for every 
$m \in M$, it holds that $\mu^{\mathrm{M}} \succeq_{m} \mu$. Similarly, for any matching $\mu$ in the core, for every $w \in W$, it holds that $\mu^{\mathrm{W}} \succeq_{w} \mu$.

\section{The Myopic-Farsighted Stable Set}

The literature on network and coalition formation can be divided into two streams, depending on whether the approach taken is myopic or farsighted. While the notions of the core and the vNM stable set assume myopic players in the sense that individual and coalitional deviations are not anticipated to be countered by subsequent deviations, the notions of farsighted core and of vNM farsightedly stable set assume farsighted players that take the moves of other coalitions into account and consider the end outcome that their deviations may lead to. ${ }^{8}$

For marriage problems, a vNM stable set contains the core according to Ehlers (2007). It exists and is unique due to results by Wako (2010). Herings, Mauleon, and Vannetelbosch (2017) argue that the standard formulation of the vNM stable set violates coalitional sovereignty and propose to replace dominance by path dominance. The resulting concept is called the $\mathrm{CP}$ vNM set and is shown to coincide with the core. Mauleon, Vannetelbosch, and Vergote (2011) characterize the vNM farsightedly stable sets as the singleton core elements.

Up to now, no solution concept has been proposed in order to allow for heterogeneity in the degree of farsightedness among players. In the following, we propose the notion of myopic-farsighted stable set to study the matchings that are stable when players can be both myopic and farsighted.

Let $F \subset N$ denote the set of farsighted players. The set $F$ is allowed to be empty. A myopic-farsighted improving path is a sequence of matchings that can emerge when farsighted players form or destroy links based on the improvement the end matching offers them relative to the current matching while myopic players form or destroy links based on the improvement the next matching in the sequence offers them relative to the current one.

Definition 1. Let $(M, W, \succ)$ be a marriage problem with set of farsighted players $F$. A myopic-farsighted improving path from a matching $\mu \in \mathcal{M}$ to a matching $\mu^{\prime} \in \mathcal{M}$

\footnotetext{
${ }^{8}$ See Chwe (1994), Xue (1998), Diamantoudi and Xue (2003), Mauleon and Vannetelbosch (2004), Page, Wooders, and Kamat (2005), Herings, Mauleon and Vannetelbosch (2004, 2009), and Page and Wooders (2009) among others.
} 
is a finite sequence of distinct matchings $\mu_{0}, \ldots, \mu_{L}$ with $\mu_{0}=\mu$ and $\mu_{L}=\mu^{\prime}$ such that for every $\ell \in\{0, \ldots, L-1\}$ either (i) or (ii) holds:

(i) $\mu_{\ell+1}=\mu_{\ell}-(m, w)$ for some $(m, w) \in M \times W$ such that

$$
\begin{cases}\mu_{\ell+1}(m) \succ_{m} \mu_{\ell}(m) & \text { if } m \in M \backslash F \\ \mu_{L}(m) \succ_{m} \mu_{\ell}(m) & \text { if } m \in F\end{cases}
$$

or

$$
\begin{cases}\mu_{\ell+1}(w) \succ_{w} \mu_{\ell}(w) & \text { if } w \in W \backslash F \\ \mu_{L}(w) \succ_{w} \mu_{\ell}(w) & \text { if } w \in F .\end{cases}
$$

(ii) $\mu_{\ell+1}=\mu_{\ell}+(m, w)$ for some $(m, w) \in M \times W$ such that

$$
\begin{cases}\mu_{\ell+1}(m) \succeq_{m} \mu_{\ell}(m) & \text { if } m \in M \backslash F \\ \mu_{L}(m) \succeq_{m} \mu_{\ell}(m) & \text { if } m \in F\end{cases}
$$

and

$$
\begin{cases}\mu_{\ell+1}(w) \succeq_{w} \mu_{\ell}(w) & \text { if } w \in W \backslash F \\ \mu_{L}(w) \succeq_{w} \mu_{\ell}(w) & \text { if } w \in F\end{cases}
$$

with at least one of these preferences being strict.

Each matching in the sequence differs from the previous one in that either an existing match in the previous matching is destroyed like in case (i) or a new match is formed between a man and a woman that are not matched to one another in the previous matching as in case (ii).

If there exists a myopic-farsighted improving path from a matching $\mu$ to a matching $\mu^{\prime}$, then we write $\mu \rightarrow \mu^{\prime}$. The set of all matchings that can be reached from a matching $\mu \in \mathcal{M}$ by a myopic-farsighted improving path is denoted by $h(\mu)$, so

$$
h(\mu)=\left\{\mu^{\prime} \in \mathcal{M} \mid \mu \rightarrow \mu^{\prime}\right\} .
$$

Example 1. Consider the marriage problem $(M, W, \succ)$, which corresponds to Example 2.31 of Roth and Sotomayor (1990) with the roles of men and women reversed. It holds that $M=\left\{m_{1}, m_{2}, m_{3}\right\}$ and $W=\left\{w_{1}, w_{2}, w_{3}\right\}$. Assume $F=\left\{w_{1}, w_{3}\right\}$, so women $w_{1}$ and $w_{3}$ are farsighted and all men and woman $w_{2}$ are myopic. The 
preferences of the players are as follows.

\begin{tabular}{|c|c|c|c|c|c|}
\hline$\underline{m_{1}}$ & $\underline{m_{2}}$ & $\underline{m_{3}}$ & $\underline{w_{1}}$ & $\underline{w_{2}}$ & $\underline{w_{3}}$ \\
\hline$w_{1}$ & $w_{3}$ & $w_{1}$ & $m_{2}$ & $m_{1}$ & $m_{1}$ \\
\hline$w_{2}$ & $w_{1}$ & $w_{2}$ & $m_{1}$ & $m_{2}$ & $m_{2}$ \\
\hline$w_{3}$ & $w_{2}$ & $w_{3}$ & $m_{3}$ & $m_{3}$ & \\
\hline
\end{tabular}

By applying the deferred acceptance algorithm of Gale and Shapley (1962), it can be easily verified that the woman-optimal stable matching is equal to

$$
\begin{aligned}
& \mu^{\mathrm{W}}\left(m_{1}\right)=w_{1}, \\
& \mu^{\mathrm{W}}\left(m_{2}\right)=w_{3}, \\
& \mu^{\mathrm{W}}\left(m_{3}\right)=w_{2} .
\end{aligned}
$$

The matching $\mu$ defined by

$$
\begin{aligned}
& \mu\left(m_{1}\right)=w_{3}, \\
& \mu\left(m_{2}\right)=w_{1}, \\
& \mu\left(m_{3}\right)=w_{2},
\end{aligned}
$$

is strictly preferred by $w_{1}$ and $w_{3}$ to $\mu^{\mathrm{W}}$ and does not make a difference for $w_{2}$. However, the pair $\left(m_{1}, w_{2}\right)$ can block $\mu$, so $\mu$ does not belong to the core.

It holds that $\mu \in h\left(\mu^{\mathrm{W}}\right)$, so it is possible that farsighted women leave the womanoptimal stable matching by means of a myopic-farsighted improving path. To see this, consider the myopic-farsighted improving path $\mu_{0}, \ldots, \mu_{4}$ with $\mu_{0}=\mu^{\mathrm{W}}$ and $\mu_{4}=\mu$, where $\mu_{1}=\mu_{0}-\left(m_{1}, w_{1}\right), \mu_{2}=\mu_{1}-\left(m_{2}, w_{3}\right), \mu_{3}=\mu_{2}+\left(m_{2}, w_{1}\right)$, and $\mu_{4}=\mu_{3}+\left(m_{1}, w_{3}\right)$. This myopic-farsighted improving path is illustrated in Figure 1.

The move to $\mu_{1}$ is initiated by $w_{1}$ who is farsighted and therefore wants to sever her link with $m_{1}$ in the anticipation of ending up in a match with $m_{2}$. Similarly, the move from $\mu_{1}$ to $\mu_{2}$ is initiated by $w_{3}$ who is farsighted and is willing to cut her link with $m_{2}$ in the expectation of being matched with $m_{1}$. The transition to $\mu_{4}=\mu$ is completed by the subsequent marriages of the single players $m_{2}$ and $w_{1}$ and the single players $m_{1}$ and $w_{3}$.

More surprising perhaps is that also $\mu^{\mathrm{W}} \in h(\mu)$. So even though two of the three women are farsighted, it is possible that they move from $\mu$ to a matching that none of them strictly prefers and that is strictly worse for women $w_{1}$ and $w_{3}$. To verify this statement, consider the myopic-farsighted improving path $\mu_{0}, \ldots, \mu_{4}$ with $\mu_{0}=\mu$ 


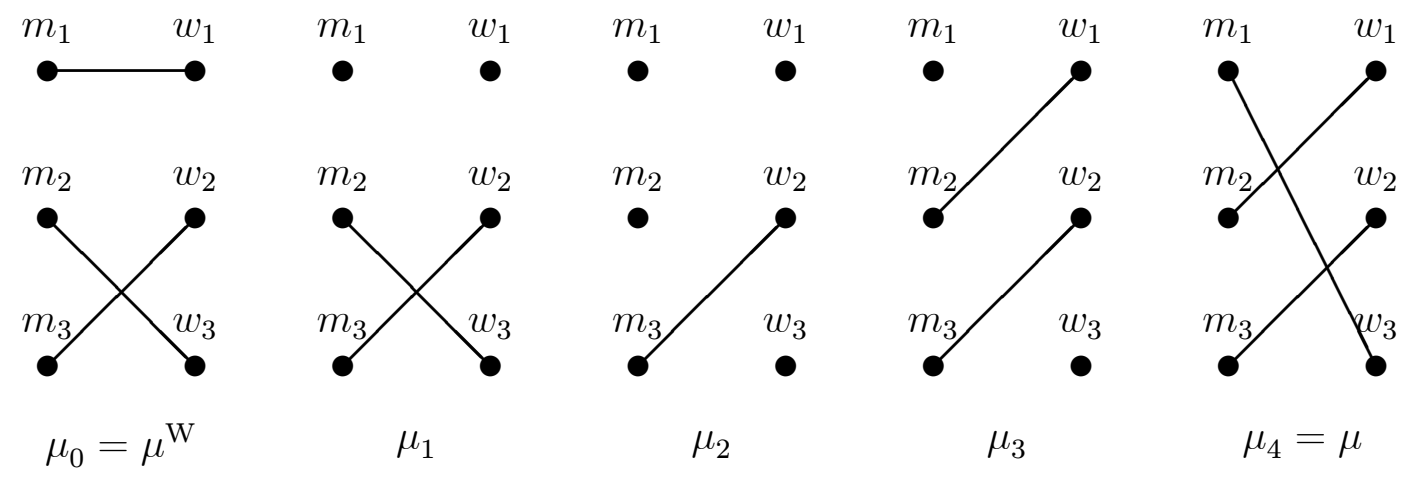

Figure 1: Myopic-farsighted improving path in Example 1 to move from $\mu^{\mathrm{W}}$ to $\mu$.

and $\mu_{4}=\mu^{\mathrm{W}}$, where $\mu_{1}=\mu_{0}+\left(m_{1}, w_{2}\right), \mu_{2}=\mu_{1}+\left(m_{2}, w_{3}\right), \mu_{3}=\mu_{2}+\left(m_{1}, w_{1}\right)$, and $\mu_{4}=\mu_{3}+\left(m_{3}, w_{2}\right)$. This myopic-farsighted improving path is illustrated in Figure 2.

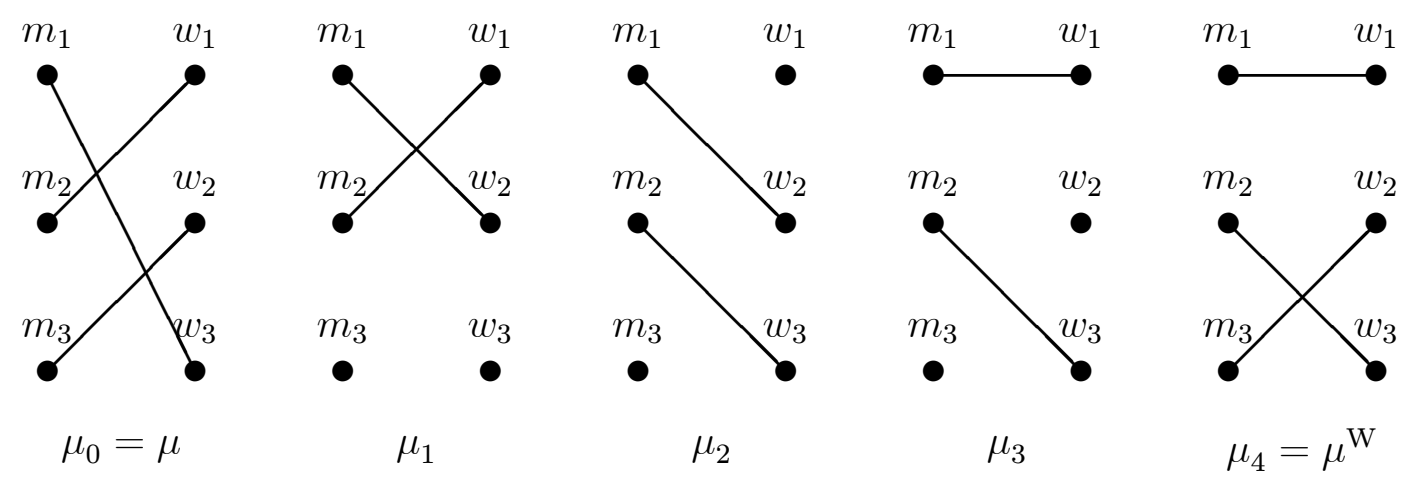

Figure 2: Myopic-farsighted improving path in Example 1 to move from $\mu$ to $\mu^{\mathrm{W}}$.

Since $m_{1}$ is myopic and $w_{2}$ is myopic, it is possible to establish a link between them as man $m_{1}$ strictly prefers $w_{2}=\mu_{1}\left(m_{1}\right)$ to $w_{3}=\mu_{0}\left(m_{1}\right)$ and woman $w_{2}$ strictly prefers $m_{1}=\mu_{1}\left(w_{2}\right)$ to $m_{3}=\mu_{0}\left(w_{2}\right)$. Since at $\mu_{1}$ woman $w_{3}$ has become single, she is willing to form a link with $m_{2}$, her partner in the end matching of the sequence, moving from $\mu_{1}$ to $\mu_{2}$. Since at $\mu_{2}$ woman $w_{1}$ is single, she is willing to team up with $m_{1}$, her partner in the end matching of the sequence, leading to the matching $\mu_{3}$. Woman $w_{2}$ has become single at $\mu_{3}$ and marries $m_{3}$ in order to move to the end matching $\mu_{4}$. The men are myopically improving in each step of the sequence.

Assume now $F=\left\{w_{1}, w_{2}, w_{3}\right\}$, so all women are farsighted whereas all men 
are myopic. It can be verified that the myopic-farsighted improving paths that were used to show that $\mu^{\mathrm{W}} \rightarrow \mu$ and $\mu \rightarrow \mu^{\mathrm{W}}$ are still valid, though the reasoning changes occasionally when it involves a move by woman $w_{2}$ who is now farsighted.

The myopic-farsighted stable set results when we replace the conditions of internal and external stability in the vNM stable set as based on direct dominance by the conditions as based on the myopic-farsighted improving paths.

Definition 2. Let $(M, W, \succ)$ be a marriage problem with set of farsighted players $F$. A set of matchings $V \subset \mathcal{M}$ is a myopic-farsighted stable set if it satisfies:

(i) Internal stability: For every $\mu, \mu^{\prime} \in V$, it holds that $\mu^{\prime} \notin h(\mu)$.

(ii) External stability: For every $\mu \in \mathcal{M} \backslash V$, it holds that $h(\mu) \cap V \neq \emptyset$.

Condition (i) of Definition 2 corresponds to internal stability. For any two matchings $\mu$ and $\mu^{\prime}$ in the myopic-farsighted stable set $V$ it does not hold that $\mu \rightarrow \mu^{\prime}$. Condition (ii) of Definition 2 expresses external stability. For every matching $\mu$ outside the myopic-farsighted stable set $V$ it holds that there is $\mu^{\prime} \in V$ such that $\mu \rightarrow \mu^{\prime}$.

In Example 2, we consider the most basic situation of conflict between the objectives of men and women, where the most preferred woman of each man ranks him as the worst possible marriage partner.

Example 2. Consider the marriage problem $(M, W, \succ)$ with two men, $M=\left\{m_{1}, m_{2}\right\}$, and two women, $W=\left\{w_{1}, w_{2}\right\}$. Assume $F=W$, so the women are farsighted and the men are myopic. The preferences of men and women are diametrically opposed to each other:

$\begin{array}{lllll}\frac{m_{1}}{w_{1}} & \frac{m_{2}}{w_{2}} & & \frac{w_{1}}{m_{2}} & \frac{w_{2}}{m_{1}} \\ w_{2} & w_{1} & & m_{1} & m_{2} .\end{array}$

There are seven possible matchings, illustrated in Figure 3. The man-optimal stable matching is equal to $\mu_{6}$ and the woman-optimal stable matching to $\mu_{7}$. Table 1 presents the matchings that can be reached from a given initial matching by means of a myopic-farsighted improving path. Notice that $\mu^{\mathrm{W}} \in h\left(\mu^{\mathrm{M}}\right)$ because the farsighted woman $w_{1}$ first leaves $m_{1}$ to become single at the matching $\mu_{4}$. Next, the farsighted 


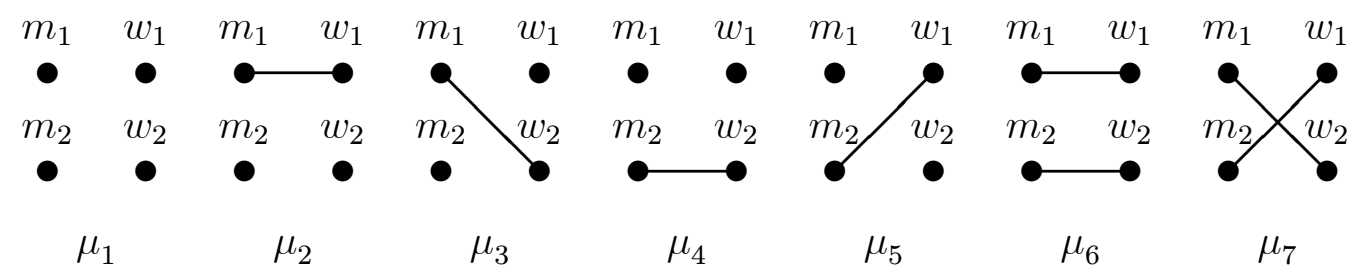

Figure 3: All possible matchings in Example 2, where $\mu_{6}=\mu^{\mathrm{M}}$ and $\mu_{7}=\mu^{\mathrm{W}}$.

\begin{tabular}{|c|c|c|c|c|c|c|}
\hline$\mu$ & \multicolumn{6}{|c|}{$h(\mu)$} \\
\hline$\mu_{1}$ & $\mu_{2}$ & $\mu_{3}$ & $\mu_{4}$ & $\mu_{5}$ & $\mu_{6}$ & $\mu_{7}$ \\
\hline$\mu_{2}$ & & & & $\mu_{5}$ & $\mu_{6}$ & $\mu_{7}$ \\
\hline$\mu_{3}$ & $\mu_{2}$ & & & $\mu_{5}$ & $\mu_{6}$ & $\mu_{7}$ \\
\hline$\mu_{4}$ & & $\mu_{3}$ & & & $\mu_{6}$ & $\mu_{7}$ \\
\hline$\mu_{5}$ & & $\mu_{3}$ & $\mu_{4}$ & & $\mu_{6}$ & $\mu_{7}$ \\
\hline$\mu_{6}$ & & & & & & $\mu_{7}$ \\
\hline$\mu_{7}$ & & $\mu_{3}$ & & $\mu_{5}$ & & \\
\hline
\end{tabular}

Table 1: The set of matchings that can be reached by a myopic-farsighted improving path in Example 2 when $F=\left\{w_{1}, w_{2}\right\}$. It holds that $\mu_{6}=\mu^{\mathrm{M}}$ and $\mu_{7}=\mu^{\mathrm{W}}$. 
woman $w_{2}$ marries $m_{1}$ to arrive at the matching $\mu_{3}$. Next, $w_{1}$ marries $m_{2}$ to reach $\mu^{\mathrm{W}}$.

We argue that the set $V=\left\{\mu^{\mathrm{W}}\right\}$ is a myopic-farsighted stable set. The condition of internal stability in Definition 2 is satisfied since the set $V$ is a singleton. Since for every $\mu \in \mathcal{M} \backslash\left\{\mu^{\mathrm{W}}\right\}$, it holds that $\mu_{7} \in h(\mu)$, the condition of external stability in Definition 2 is satisfied as well. We have shown that $V=\left\{\mu^{\mathrm{W}}\right\}$ is a myopicfarsighted stable set.

It is not hard to demonstrate that there are no other myopic-farsighted stable sets. Let $V$ be a myopic-farsighted stable set not equal to $\left\{\mu^{\mathrm{W}}\right\}$. The condition of internal stability in Definition 2 together with the fact that $\mu^{\mathrm{W}} \in h(\mu)$ for every $\mu \in \mathcal{M} \backslash\left\{\mu^{\mathrm{W}}\right\}$ implies that

$$
\mu^{\mathrm{W}} \notin V
$$

To satisfy the condition of external stability in Definition 2, it should therefore hold that

$$
\mu_{3} \in V \text { or } \mu_{5} \in V
$$

Since $h\left(\mu_{6}\right)=\left\{\mu_{7}\right\}$ and $\mu_{7} \notin V$, external stability implies that

$$
\mu_{6} \in V
$$

Since $\mu_{6} \in h\left(\mu_{3}\right)$ and $\mu_{6} \in h\left(\mu_{5}\right)$, we obtain a contradiction with internal stability.

We next analyze the case in which exactly one player is farsighted, say woman $w_{1}$. Table 1 remains almost unchanged, except that it is no longer the case that $\mu_{3}$ belongs to $h\left(\mu_{7}\right)$. The argument that $V=\left\{\mu_{7}\right\}$ is a myopic-farsighted stable set remains unaffected. The argument that there is no other myopic-farsighted stable set proceeds along the same lines as before and becomes slightly easier. The other three cases with only only farsighted player follow by symmetry.

Example 2 shows that in the most basic situation of conflict between the objectives of men and women, farsighted women are able to obtain their most preferred solution. Moreover, this is the unique prediction as made by the concept of the myopic-farsighted stable set. In fact, it is not even needed that both women are farsighted. Even if only one of them is farsighted, the woman-optimal stable matching results as the unique prediction. 
Example 3. We take the same primitives as in Example 2, but now vary the assumptions with respect to farsightedness.

The case where nobody is farsighted leads to a concept that is equivalent to the pairwise CP vNM set of Herings, Mauleon, and Vannetelbosch (2017), see also Section 4 . It follows from their Theorem 1 that the core is the unique myopic-farsighted stable set, so $V=\left\{\mu^{\mathrm{M}}, \mu^{\mathrm{W}}\right\}$. The main intuition for this result comes from the contribution by Roth and Vande Vate (1990), who have shown that it is possible to reach a core element from any initial matching by a sequence of myopic improvements, and the fact that at a core element myopic improvements are impossible.

We now turn to the case where all players are farsighted, $F=M \cup W$. Table 2 presents the matchings that can be reached from a given initial matching by means of a myopic-farsighted improving path. Since both $\mu_{6}$ and $\mu_{7}$ can be reached from

\begin{tabular}{|c|c|c|c|c|c|c|}
\hline$\mu$ & \multicolumn{6}{|c|}{$h(\mu)$} \\
\hline$\mu_{1}$ & $\mu_{2}$ & $\mu_{3}$ & $\mu_{4}$ & $\mu_{5}$ & $\mu_{6}$ & $\mu_{7}$ \\
\hline$\mu_{2}$ & & & & $\mu_{5}$ & $\mu_{6}$ & $\mu_{7}$ \\
\hline$\mu_{3}$ & $\mu_{2}$ & & & & $\mu_{6}$ & $\mu_{7}$ \\
\hline$\mu_{4}$ & & $\mu_{3}$ & & & $\mu_{6}$ & $\mu_{7}$ \\
\hline$\mu_{5}$ & & & $\mu_{4}$ & & $\mu_{6}$ & $\mu_{7}$ \\
\hline$\mu_{6}$ & & & & & & $\mu_{7}$ \\
\hline$\mu_{7}$ & & & & & $\mu_{6}$ & \\
\hline
\end{tabular}

Table 2: The set of matchings that can be reached by a myopic-farsighted improving path in Example 3 when $F=M \cup W$. It holds that $\mu_{6}=\mu^{\mathrm{M}}$ and $\mu_{7}=\mu^{\mathrm{W}}$.

any other matching, it follows that both $\left\{\mu_{6}\right\}$ and $\left\{\mu_{7}\right\}$ are myopic-farsighted stable sets. It is easily verified that there are no other myopic-farsighted stable sets. As in the case of completely myopic players, we obtain all core elements as the unique prediction, be it that these elements are predicted as singletons in the farsighted case.

The case where all players are farsighted with the exception of one player, say $F=\left\{m_{1}, w_{1}, w_{2}\right\}$, is very close to the situation where everyone is farsighted. Compared to Table 2 , the only change is that $\mu_{3} \in h\left(\mu_{5}\right)$ and $\mu_{3} \in h\left(\mu_{7}\right)$. This will not affect the analysis and the conclusion that the woman-optimal stable matching and the man-optimal stable matching can be both sustained as singleton myopic- 
farsighted stable sets remains.

The final case is where one player on each side is farsighted, say $F=\left\{m_{1}, w_{1}\right\}$. Table 3 presents the matchings that can be reached from a given initial matching by means of a myopic-farsighted improving path. Since both $\mu_{6}$ and $\mu_{7}$ can be reached

\begin{tabular}{|c||cccccc|}
\hline$\mu$ & \multicolumn{1}{|c|}{$h(\mu)$} \\
\hline \hline$\mu_{1}$ & $\mu_{2}$ & $\mu_{3}$ & $\mu_{4}$ & $\mu_{5}$ & $\mu_{6}$ & $\mu_{7}$ \\
$\mu_{2}$ & & & & $\mu_{5}$ & $\mu_{6}$ & $\mu_{7}$ \\
$\mu_{3}$ & $\mu_{2}$ & & & & $\mu_{6}$ & $\mu_{7}$ \\
$\mu_{4}$ & $\mu_{2}$ & $\mu_{3}$ & & & $\mu_{6}$ & $\mu_{7}$ \\
$\mu_{5}$ & $\mu_{2}$ & $\mu_{3}$ & $\mu_{4}$ & & $\mu_{6}$ & $\mu_{7}$ \\
$\mu_{6}$ & & & & & & $\mu_{7}$ \\
$\mu_{7}$ & $\mu_{2}$ & & & & $\mu_{6}$ & \\
\hline
\end{tabular}

Table 3: The set of matchings that can be reached by a myopic-farsighted improving path in Example 3 when $F=\left\{m_{1}, w_{1}\right\}$. It holds that $\mu_{6}=\mu^{\mathrm{M}}$ and $\mu_{7}=\mu^{\mathrm{W}}$.

from any other matching, it follows that both $\left\{\mu_{6}\right\}$ and $\left\{\mu_{7}\right\}$ are myopic-farsighted stable sets. It is easily verified that there are no other myopic-farsighted stable sets. The predictions are therefore identical to the case where all players are farsighted. It follows from a symmetry argument that all other cases with exactly one player on each side being farsighted lead to the same predictions.

Example 3 illustrates that any core element can be sustained in some myopicfarsighted stable set in case both sides of the markets are similar in terms of their degree of farsightedness as well as in the case where all players are farsighted except one.

\section{Homogeneous Societies}

In this section we consider the case where either all players are myopic or all players are farsighted.

First consider the case where all players are myopic, so $F=\emptyset$. Definition 2 then boils down to the pairwise CP vNM set as defined in Herings, Mauleon, and Vannetelbosch (2017). This set differs from the standard notion of a vNM set in 
three important ways. The standard definition, see Ehlers (2007) and Wako (2010), violates the assumption of coalitional sovereignty, the property that an objecting coalition cannot enforce the organization of players outside the coalition. Second, the standard definition of the vNM set is such that it does not take into account that a deviation by a coalition can be followed by further deviations. The pairwise CP vNM set follows the approach by van Deemen (1991) and Page and Wooders (2009), which takes into account that if a matching is blocked by some coalition and the resulting matching is not in the stable set itself, then further deviations will take place. This observation leads van Deemen (1991) to define the generalized stable set for abstract systems and Page and Wooders (2009) to define the stable set with respect to path dominance. Third, we restrict ourselves to deviations by single players and pairs. It follows from the results in Herings, Mauleon, and Vannetelbosch (2017) that identical results are obtained when coalitions of arbitrary size are allowed to move.

The following result is stated as Theorem 1 in Herings, Mauleon, and Vannetelbosch (2017). The proof is based on a result by Roth and Vande Vate (1990), claiming that, from any matching that does not belong to the core, a core element can be reached by a finite sequence of myopic improvements.

Theorem 1. Let $(M, W, \succ)$ be a marriage problem with set of farsighted players $F=\emptyset$. A set of matchings is a myopic-farsighted stable set if and only if it is equal to the core.

At the other side of the spectrum, we have the case where all players are farsighted, so $F=M \cup W$. Definition 2 is then closely related to the vNM farsightedly stable set as defined in Mauleon, Vannetelbosch, and Vergote (2011), which in turn is based on the work by Harsanyi (1974), Chwe (1994), and Diamantoudi and Xue (2003). The only difference is that we restrict ourselves to deviations by single players and pairs. It is not hard to see that any individually rational matching that can be reached by arbitrary coalitional deviations can also be reached by deviations by single players and pairs.

The next lemma shows that any matching in a myopic-farsighted stable set in case $F=M \cup W$ is individually rational.

Lemma 1. Let $(M, W, \succ)$ be a marriage problem with set of farsighted players $F=$ $M \cup W$. Let $V$ be a myopic-farsighted stable set and $\mu \in V$. Then $\mu$ is individually 
rational.

Proof. Suppose $\mu$ is not individually rational. Then there is $m \in M$ and $w \in W$ such that $\mu(m)=w$, and $m \succ_{m} w$ or $w \succ_{w} m$. Without loss of generality, assume $m \succ_{m} w$. It holds that $\mu \notin h(\mu-(m, w))$ as the farsighted man $m$ will never accept a match with $w$. On the other hand, $\mu-(m, w) \in h(\mu)$, so by internal stability of $V$ it holds that $\mu-(m, w) \notin V$. By external stability of $V$ it holds that there is $\mu^{\prime} \in h(\mu-(m, w))$ such that $\mu^{\prime} \in V$. Let $\mu_{1}, \ldots, \mu_{L}$ with $\mu_{1}=\mu-(m, w)$ and $\mu_{L}=\mu^{\prime}$ be a myopic-farsighted improving path from $\mu-(m, w)$ to $\mu^{\prime}$. Since $m$ is farsighted, it holds that $\mu^{\prime}(m) \succeq_{m} m$. Now it follows that $\mu_{0}, \mu_{1}, \ldots, \mu_{L}$ with $\mu_{0}=\mu$ is a myopic-farsighted improving path from $\mu$ to $\mu^{\prime}$ and therefore $\mu^{\prime} \in h(\mu)$. This contradicts the fact that $V$ is internally stable. Consequently, $\mu$ is individually rational.

Mauleon, Vannetelbosch, and Vergote (2011) show that the vNM farsightedly stable sets are characterized as all singletons that consist of a core element. The next result confirms that the same characterization applies to the myopic-farsighted stable set when all players are farsighted.

Theorem 2. Let $(M, W, \succ)$ be a marriage problem with set of farsighted players $F=M \cup W$. A set of matchings is a myopic-farsighted stable set if and only if it is a singleton consisting of a core element.

Proof. Let $V$ be a myopic-farsighted stable set. By Lemma 1, every matching in $V$ is individually rational. So even when arbitrary coalitions are allowed to move, the set $V$ satisfies internal stability, and obviously also external stability. It is therefore a vNM farsightedly stable set and Theorem 2 of Mauleon, Vannetelbosch, and Vergote (2011) now implies that it is a singleton consisting of a core element.

Let $V$ be a singleton consisting of a core element. Theorem 1 of Mauleon, Vannetelbosch, and Vergote (2011) states that $V$ is a vNM farsightedly stable set, so it satisfies internal and external stability as based on arbitrary coalitional moves. Since a core element is individually rational, even when only moves by single players and pairs of players are allowed, $V$ remains to satisfy external stability, and obviously also internal stability. It follows that $V$ is a myopic-farsighted stable set.

Theorem 2 demonstrates that all core elements can be sustained when all players are farsighted, but any other matching not. 


\section{$5 \quad$ All Men Are Myopic}

Typical results in the matching literature point towards the core as the set of reasonable outcomes, but are not able to discriminate among different core elements. Does the introduction of heterogeneity in terms of farsightedness allow us to discriminate between different core elements? A closely related issue is whether farsighted players are able to enforce their optimal stable matching. For instance, is it always possible to reach the woman-optimal stable matching $\mu^{\mathrm{W}}$ from any matching $\mu \neq \mu^{\mathrm{W}}$ by means of a myopic-farsighted stable path as was the case in Example 2? The answer is affirmative under certain conditions.

In this section we study the case where the players on one side, the men, are all myopic, whereas any player on the other side, the women, can be either myopic or farsighted. For every $m \in M$, let $w^{*}(m) \in W \cup\{m\}$ denote the top choice of $m$, so $w^{*}(m) \succeq_{m} w$ for every $w \in W$ and $w^{*}(m) \succeq_{m} m$.

Assumption 1. For every $m \in M$, it holds that $w^{*}(m) \in F \cup\{m\}$.

Assumption 1 requires the top choice of every man to be a farsighted woman or to remain single. Intuitively this corresponds to the requirement that the farsighted side is desirable. It is automatically satisfied when all women are farsighted.

We prove first that the woman-optimal stable matching $\mu^{\mathrm{W}}$ can be reached from any matching $\mu$ with the property that, for every $w \in W, \mu^{\mathrm{W}}(w) \succeq_{w} \mu(w)$. Since the core has a lattice structure and the woman-optimal stable matching $\mu^{\mathrm{W}}$ is weakly preferred by all women to any other core element, Lemma 2 covers all matchings $\mu$ that belong to the core.

Lemma 2. Let $(M, W, \succ)$ be a marriage problem satisfying Assumption 1 with set of farsighted players $F \subset W$. For every $\mu \in \mathcal{M} \backslash\left\{\mu^{\mathrm{W}}\right\}$ such that, for every $w \in W$, $\mu^{\mathrm{W}}(w) \succeq_{w} \mu(w)$ it holds that $\mu^{\mathrm{W}} \in h(\mu)$.

Proof. Let $\mu \in \mathcal{M} \backslash\left\{\mu^{\mathrm{W}}\right\}$ be a matching such that, for every $w \in W, \mu^{\mathrm{W}}(w) \succeq_{w}$ $\mu(w)$. We construct a myopic-farsighted improving path $\mu_{0}, \ldots, \mu_{L}$ from $\mu_{0}=\mu$ to $\mu_{L}=\mu^{\mathrm{W}}$. Let

$$
W^{1}=\left\{w \in W \backslash F \mid \mu^{\mathrm{W}}(w) \succ_{w} \mu(w) \text { and } \mu(w) \in M\right\}
$$

be the, possibly empty, set of myopic women $w$ who strictly prefer $\mu^{\mathrm{W}}(w)$ to $\mu(w)$ and who are not single at $\mu$. Let $k^{1}$ be the cardinality of $W^{1}$. The set of men married 
at $\mu$ to a woman $w \in W^{1}$ is denoted by $M^{1}=\mu\left(W^{1}\right)$. Take an arbitrary order of the men in $M^{1}$, say $m_{0}, \ldots, m_{k^{1}-1}$.

For $\ell \in\left\{0, \ldots, k^{1}-1\right\}$, we define the matching $\mu_{\ell+1}=\mu_{\ell}+\left(m_{\ell}, w^{*}\left(m_{\ell}\right)\right)$, so the $k^{1}$ men in the set $M^{1}$ sequentially marry their top choices. We argue that the sequence of matchings $\mu_{0}, \ldots, \mu_{k^{1}}$ is the first part of a myopic-farsighted improving path from $\mu$ to $\mu^{\mathrm{W}}$ by showing that for every $\ell \in\left\{0, \ldots, k^{1}-1\right\}$ we have

(i) $\mu_{\ell+1}\left(m_{\ell}\right)=w^{*}\left(m_{\ell}\right) \succ_{m_{\ell}} \mu_{\ell}\left(m_{\ell}\right)$,

(ii) $\mu_{L}\left(w^{*}\left(m_{\ell}\right)\right)=\mu^{\mathrm{W}}\left(w^{*}\left(m_{\ell}\right)\right) \succeq_{w^{*}\left(m_{\ell}\right)} \mu_{\ell}\left(w^{*}\left(m_{\ell}\right)\right)$.

Let some $\ell \in 0, \ldots, k^{1}-1$ be given. The strict preference in (i) holds because $m_{\ell}$ is married to the myopic woman $\mu_{\ell}\left(m_{\ell}\right) \in W \backslash F$, whereas his top choice is a farsighted woman.

We now show that (ii) holds. If $\mu_{\ell}\left(w^{*}\left(m_{\ell}\right)\right)=\mu\left(w^{*}\left(m_{\ell}\right)\right)$, then it holds that $\mu^{\mathrm{W}}\left(w^{*}\left(m_{\ell}\right)\right) \succeq_{w^{*}\left(m_{\ell}\right)} \mu_{\ell}\left(w^{*}\left(m_{\ell}\right)\right)$ by assumption. Otherwise, there is $\ell^{\prime}<\ell$ such that

$$
w^{*}\left(m_{\ell^{\prime}}\right)=w^{*}\left(m_{\ell}\right) \text { and } \mu_{\ell}\left(w^{*}\left(m_{\ell}\right)\right)=\mu_{\ell^{\prime}}\left(w^{*}\left(m_{\ell^{\prime}}\right)\right)=m_{\ell^{\prime}} .
$$

Suppose $m_{\ell^{\prime}} \succ_{w^{*}\left(m_{\ell^{\prime}}\right)} \mu^{\mathrm{W}}\left(w^{*}\left(m_{\ell^{\prime}}\right)\right)$. Then $\mu^{\mathrm{W}}\left(m_{\ell^{\prime}}\right) \neq w^{*}\left(m_{\ell^{\prime}}\right)$, so it follows that $w^{*}\left(m_{\ell^{\prime}}\right) \succ_{m_{\ell^{\prime}}} \mu^{\mathrm{W}}\left(m_{\ell^{\prime}}\right)$. Now the pair $\left(m_{\ell^{\prime}}, w^{*}\left(m_{\ell^{\prime}}\right)\right)$ can block $\mu^{\mathrm{W}}$, a contradiction. Consequently, it holds that $\mu^{\mathrm{W}}\left(w^{*}\left(m_{\ell^{\prime}}\right)\right) \succeq_{w^{*}\left(m_{\ell^{\prime}}\right)} m_{\ell^{\prime}}$, which is equivalent to $\mu^{\mathrm{W}}\left(w^{*}\left(m_{\ell}\right)\right) \succeq_{w^{*}\left(m_{\ell}\right)} \mu_{\ell}\left(w^{*}\left(m_{\ell}\right)\right)$, so (ii) holds.

At $\mu_{k^{1}}$, every man in $M^{1}$ is either single or married to his top choice.

Let

$$
M^{2}=\left\{m \in M \mid \mu_{k^{1}}(m) \in F \backslash\left\{\mu^{\mathrm{W}}(m)\right\}\right\}
$$

be the, possibly empty, set of men that are married at $\mu_{k^{1}}$ to a farsighted woman different from $\mu^{\mathrm{W}}(m)$. For every $m \in M^{2}$ it holds that either $m \in M^{1}$ and $\mu_{k^{1}}(m)=$ $w^{*}(m) \neq \mu^{\mathrm{W}}(m)$, or $m \in M \backslash M^{1}$ and $\mu_{k^{1}}(m) \in F$ is such that $\mu^{\mathrm{W}}\left(\mu_{k^{1}}(m)\right) \succ_{\mu_{k^{1}}(m)}$ $m$, where in the latter case we use that $\mu_{k^{1}}(m)=\mu(m)$. Let $k^{2}$ be the cardinality of the set $M^{2}$. Take an arbitrary order of the men in $M^{2}$, say $m_{0}, \ldots, m_{k^{2}-1}$.

For $\ell \in\left\{0, \ldots, k^{2}-1\right\}$, we define the matching $\mu_{k^{1}+\ell+1}=\mu_{k^{1}+\ell}-\left(m_{\ell}, \mu_{k^{1}+\ell}\left(m_{\ell}\right)\right)$, so the $k^{2}$ farsighted women married to the men in $M^{2}$ sequentially destroy their matches under $\mu_{k^{1}}$ and all men in $M^{2}$ become single.

We argue that the sequence of matchings $\mu_{k^{1}}, \ldots, \mu_{k^{1}+k^{2}}$ is the second part of a myopic-farsighted improving path from $\mu$ to $\mu^{\mathrm{W}}$ by showing that, for every $\ell \in$ $\left\{0, \ldots, k^{2}-1\right\}, \mu^{\mathrm{W}}\left(\mu_{k^{1}+\ell}\left(m_{\ell}\right)\right) \succ_{\mu_{k}+\ell}\left(m_{\ell}\right) m_{\ell}$. 
Let some $\ell \in\left\{0, \ldots, k^{2}-1\right\}$ be given. If $m_{\ell} \in M \backslash M^{1}$, then the assertion above is obviously true, so consider the case $m \in M^{1}$. Suppose $m_{\ell} \succeq_{\mu_{k^{1}+\ell}\left(m_{\ell}\right)} \mu^{\mathrm{W}}\left(\mu_{k^{1}+\ell}\left(m_{\ell}\right)\right)$. Since $\mu_{k^{1}+\ell}\left(m_{\ell}\right)=w^{*}\left(m_{\ell}\right) \neq \mu^{\mathrm{W}}\left(m_{\ell}\right)$ by construction of $M^{2}$, we have $m_{\ell} \succ_{w^{*}\left(m_{\ell}\right)}$ $\mu^{\mathrm{W}}\left(w^{*}\left(m_{\ell}\right)\right)$ and $w^{*}\left(m_{\ell}\right) \succ_{m_{\ell}} \mu^{\mathrm{W}}\left(m_{\ell}\right)$, so the pair $\left(m_{\ell}, w^{*}\left(m_{\ell}\right)\right)$ blocks $\mu^{\mathrm{W}}$, a contradiction. Consequently, it holds that $\mu^{\mathrm{W}}\left(\mu_{k^{1}+\ell}\left(m_{\ell}\right)\right) \succ_{\mu_{k^{1}+\ell}\left(m_{\ell}\right)} m_{\ell}$.

At $\mu_{k^{1}+k^{2}}$, every man $m \in M^{1}$ is single or matched to $\mu^{\mathrm{W}}(m)$. Also, every man $m \in M \backslash M^{1}$ is single or matched to $\mu^{\mathrm{W}}(m)$.

Let

$$
M^{3}=\left\{m \in M \mid \mu_{k^{1}+k^{2}}(m)=m \text { and } \mu^{\mathrm{W}}(m) \in W\right\}
$$

be the, possibly empty, set of men that are single at $\mu_{k^{1}+k^{2}}$ and married at $\mu^{\mathrm{W}}$. Let

$$
W^{3}=\left\{w \in W \mid \mu_{k^{1}+k^{2}}(w)=w \text { and } \mu^{\mathrm{W}}(w) \in M\right\}
$$

be the, possibly empty, set of women that are single at $\mu_{k^{1}+k^{2}}$ and married at $\mu^{\mathrm{W}}$. Let $k^{3}=\left|M^{3}\right|=\left|W^{3}\right|$ be the cardinality of these sets. Take an arbitrary order of the men in $M^{3}$, say $m_{0}, \ldots, m_{k^{3}-1}$.

For $\ell \in\left\{0, \ldots, k^{3}-1\right\}$, we define $\mu_{k^{1}+k^{2}+\ell+1}=\mu_{k^{1}+k^{2}+\ell}+\left(m_{\ell}, \mu^{\mathrm{W}}\left(m_{\ell}\right)\right)$, so the men in $M^{3}$ sequentially marry the women in $W^{3}$ to whom they are matched under $\mu^{\mathrm{W}}$ until we arrive at $\mu_{k^{1}+k^{2}+k^{3}}=\mu^{\mathrm{W}}$. It holds that

$$
\begin{aligned}
\mu_{k^{1}+k^{2}+\ell+1}\left(m_{\ell}\right) & =\mu^{\mathrm{W}}\left(m_{\ell}\right) \succ_{m_{\ell}} m_{\ell}=\mu_{k^{1}+k^{2}+\ell}\left(m_{\ell}\right), & & \\
\mu_{k^{1}+k^{2}+\ell+1}\left(w_{\ell}\right) & =\mu^{\mathrm{W}}\left(w_{\ell}\right) \succ_{w_{\ell}} w_{\ell}=\mu_{k^{1}+k^{2}+\ell}\left(w_{\ell}\right), & & \text { if } w_{\ell} \in W \backslash F, \\
\mu_{k^{1}+k^{2}+k^{3}}\left(w_{\ell}\right) & =\mu^{\mathrm{W}}\left(w_{\ell}\right) \succ_{w_{\ell}} w_{\ell}=\mu_{k^{1}+k^{2}+\ell}\left(w_{\ell}\right), & & \text { if } w_{\ell} \in F,
\end{aligned}
$$

so the conditions in Definition 1 are satisfied.

We prove Lemma 2 using the fact that any woman $w \in W$ either strictly prefers $\mu^{\mathrm{W}}$ to $\mu \in \mathcal{M} \backslash\left\{\mu^{\mathrm{W}}\right\}$ or is indifferent between $\mu$ and $\mu^{\mathrm{W}}$. We first identify the set $W^{1}$ of myopic women that strictly prefer $\mu^{\mathrm{W}}$ to $\mu$ and that are not single at $\mu$, together with the set $M^{1}$ of men married at $\mu$ to a woman in $W^{1}$. In order to reach $\mu^{\mathrm{W}}$ departing from $\mu$, we first allow each man in $M^{1}$ to marry his top choice and we show that the top choice weakly prefers the end matching $\mu^{\mathrm{W}}$ to the current matching. Next, we let each farsighted woman that is matched to a man different from the one at $\mu^{\mathrm{W}}$ destroy her match and become single and we show that each of these farsighted women strictly prefer the end matching $\mu^{\mathrm{W}}$ to the current matching. 
Finally, we let all single men that are married at $\mu^{\mathrm{W}}$ form the corresponding match. Thus, we have constructed a myopic-farsighted improving path from $\mu$ to $\mu^{\mathrm{W}}$.

The next lemma is known as the blocking lemma and is due to J.S. Hwang. It is presented as Lemma 3.5 in Roth and Sotomayor (1990). For an arbitrary matching $\mu \in \mathcal{M}$, we define the set of women that strictly prefer $\mu$ to $\mu^{\mathrm{W}}$ by $W(\mu)$, so

$$
W(\mu)=\left\{w \in W \mid \mu(w) \succ_{w} \mu^{\mathrm{W}}(w)\right\}
$$

It follows from the lattice structure of the core that if $\mu \in C$, then we have $W(\mu)=\emptyset$.

Lemma 3. Let $(M, W, \succ)$ be a marriage problem and let $\mu \in \mathcal{M}$ be an individually rational matching. If $W(\mu)$ is non-empty, then there is a pair $(m, w) \in \mu(W(\mu)) \times$ $(W \backslash W(\mu))$ that blocks $\mu$.

Lemma 3 states that if the set of women that strictly prefer the individually rational matching $\mu$ to $\mu^{\mathrm{W}}$ is non-empty, then there is a blocking pair $(m, w)$ such that $m$ is married to a woman strictly preferring $\mu$ and $w$ is weakly preferring $\mu^{\mathrm{W}}$. Lemma 3 is crucial for the proof of Lemma 4, which complements the case studied in Lemma 2.

Lemma 4. Let $(M, W, \succ)$ be a marriage problem satisfying Assumption 1 with set of farsighted players $F \subset W$. For every $\mu \in \mathcal{M}$ such that $W(\mu) \neq \emptyset$ it holds that $\mu^{\mathrm{W}} \in h(\mu)$.

Proof. We construct a myopic-farsighted improving path $\mu_{0}, \ldots, \mu_{L}$ from $\mu_{0}=\mu$ to $\mu_{L}=\mu^{\mathrm{W}}$. Let

$$
W^{1}=\left\{w \in \mu(M) \mid \mu(w) \succ_{\mu(w)} w \text { or } w \succ_{w} \mu(w)\right\}
$$

be the, possibly empty, set of women that are involved in a match that is not individually rational for at least one of the partners involved and denote the cardinality of $W^{1}$ by $k^{1}$. Take an arbitrary order of the women in $W^{1}$, say $w_{0}, \ldots, w_{k^{1}-1}$. For $\ell \in\left\{0, \ldots, k^{1}-1\right\}$, we define the matching $\mu_{\ell+1}=\mu_{\ell}-\left(\mu\left(w_{\ell}\right), w_{\ell}\right)$, so the player who is involved in a match under $\mu$ that is not individually rational destroys his or her link.

Consider the set $W\left(\mu_{k^{1}}\right)$ of women that strictly prefer $\mu_{k^{1}}(w)$ to $\mu^{\mathrm{W}}(w)$. The set $W\left(\mu_{k^{1}}\right)$ is empty if and only if all women in $W(\mu)$ were matched at $\mu$ to a man that preferred to be single. For $\ell \in\left\{k^{1}, k^{1}+1, \ldots\right\}$, whenever $W\left(\mu_{\ell}\right) \neq \emptyset$, select 
some $\left(m_{\ell}, w_{\ell}\right) \in \mu_{\ell}\left(W\left(\mu_{\ell}\right)\right) \times\left(W \backslash W\left(\mu_{\ell}\right)\right)$ that blocks $\mu_{\ell}$. Such a pair $\left(m_{\ell}, w_{\ell}\right)$ is guaranteed to exist by Lemma 3 . We define the matching $\mu_{\ell+1}=\mu_{\ell}+\left(m_{\ell}, w_{\ell}\right)$.

We argue next that after a finite number of steps, say $k^{2}$, the set $W\left(\mu_{k^{1}+k^{2}}\right)=\emptyset$. Since for every $\ell \geq k^{1}$, the man involved in the block is married, it follows that the cardinality of the set $\mu_{\ell}\left(W\left(\mu_{\ell}\right)\right)$ of men married to women in $W\left(\mu_{\ell}\right)$ is weakly decreasing in $\ell$ and that these sets are nested in one another. The only possibility for the cardinality of this set to remain the same is that woman $w_{\ell}$ is single under $\mu_{\ell}$. In that case, it holds that $\mu_{\ell+1}\left(m_{\ell}\right)=w_{\ell} \succ_{m_{\ell}} \mu_{\ell}\left(m_{\ell}\right)$ and, for every $m \in$ $\mu_{\ell}\left(W\left(\mu_{\ell}\right)\right) \backslash\left\{m_{\ell}\right\}, \mu_{\ell+1}(m)=\mu_{\ell}(m)$. Man $m_{\ell}$ is strictly improving and all other men in $\mu_{\ell}\left(W\left(\mu_{\ell}\right)\right)$ remain married to the same partner. It follows that cycling is impossible, so after a finite number of steps $k^{2}$ we have $W\left(\mu_{k^{1}+k^{2}}\right)=\emptyset$.

Since $W\left(\mu_{k^{1}+k^{2}}\right)=\emptyset$, the matching $\mu_{k^{1}+k^{2}}$ is either equal to $\mu^{\mathrm{W}}$ or satisfies the assumptions of Lemma 2. In the former case we are done, in the latter case we proceed as in the proof of Lemma 2 to complete the construction of the myopicfarsighted improving path leading to $\mu^{\mathrm{W}}$. It remains to be verified that for every $\ell \in\left\{0, \ldots, k^{1}+k^{2}-1\right\}$ the conditions of Definition 1 are satisfied.

Consider some $\ell \in\left\{0, \ldots, k^{1}-1\right\}$ and let $\left(m_{\ell}, w_{\ell}\right)$ be such that $\mu_{\ell+1}=\mu_{\ell}-$ $\left(m_{\ell}, w_{\ell}\right)$. It holds that $m_{\ell} \succ_{m_{\ell}} \mu_{\ell}\left(m_{\ell}\right)$ or $w_{\ell} \succ_{w_{\ell}} \mu_{\ell}\left(w_{\ell}\right)$. In the former case, we have that

$$
\mu_{\ell+1}\left(m_{\ell}\right)=m_{\ell} \succ_{m_{\ell}} \mu_{\ell}\left(m_{\ell}\right),
$$

and in the latter case that

$$
\begin{aligned}
\mu_{\ell+1}\left(w_{\ell}\right) & =w_{\ell} \succ_{w_{\ell}} \mu_{\ell}\left(w_{\ell}\right), & & \text { if } w_{\ell} \in W \backslash F, \\
\mu_{L}\left(w_{L}\right) & =\mu^{\mathrm{W}}\left(w_{\ell}\right) \succeq_{w_{\ell}} w_{\ell} \succ_{w_{\ell}} \mu_{\ell}\left(w_{\ell}\right), & & \text { if } w_{\ell} \in F,
\end{aligned}
$$

so the conditions of Definition 1 are satisfied.

Consider some $\ell \in\left\{k^{1}, \ldots, k^{1}+k^{2}-1\right\}$. Since $\left(m_{\ell}, w_{\ell}\right)$ blocks $\mu_{\ell}$, it follows that

$$
\mu_{\ell+1}\left(m_{\ell}\right) \succ_{m_{\ell}} \mu_{\ell}\left(m_{\ell}\right) \text { and } \mu_{\ell+1}\left(w_{\ell}\right) \succ_{w_{\ell}} \mu_{\ell}\left(w_{\ell}\right) .
$$

It holds that $w_{\ell} \in W \backslash W\left(\mu_{\ell}\right)$, so

$$
\mu^{\mathrm{W}}\left(w_{\ell}\right) \succeq_{w_{\ell}} \mu_{\ell}\left(w_{\ell}\right)
$$

Irrespective of whether $w_{\ell}$ is farsighted or myopic, the conditions of Definition 1 are therefore satisfied. 
In the proof of Lemma 4 , in order to reach $\mu^{\mathrm{W}}$ departing from $\mu$, first the matches that are not individually rational at $\mu$ are destroyed until we arrive at an individually rational matching $\mu_{k^{1}}$. Starting from $\mu_{k^{1}}$, we generate a sequence of blocking pairs $\left(m_{\ell}, w_{\ell}\right)$ with $\ell \geq k^{1}$ such that the pair $\left(m_{\ell}, w_{\ell}\right)$ blocks $\mu_{\ell}$. The blocking pair $\left(m_{\ell}, w_{\ell}\right)$ is chosen such that $m_{\ell}$ is matched at $\mu_{\ell}$ to a woman in $W\left(\mu_{\ell}\right)$, so a woman that strictly prefers $\mu_{\ell}$ to $\mu^{\mathrm{W}}$, and $w_{\ell}$ does not belong to $W\left(\mu_{\ell}\right)$. Lemma 3 guarantees that such a blocking pair exists. We show that after a finite number of steps, we arrive at a matching $\mu_{k^{1}+k^{2}}$ such that no woman strictly prefers $\mu_{k^{1}+k^{2}}$ to $\mu^{\mathrm{W}}$, i.e., $W\left(\mu_{k^{1}+k^{2}}\right)=\emptyset$. Thus, the matching $\mu_{k^{1}+k^{2}}$ either coincides with $\mu^{\mathrm{W}}$ or satisfies the assumptions of Lemma 2. In the latter case, we complete the myopic-farsighted improving path leading to $\mu^{\mathrm{W}}$ as in the proof of Lemma 2 .

From Lemmas 2 and 4 , it follows easily that $\left\{\mu^{\mathrm{W}}\right\}$ is a myopic-farsighted stable set.

Theorem 3. Let $(M, W, \succ)$ be a marriage problem satisfying Assumption 1 with set of farsighted players $F \subset W$. Then $\left\{\mu^{\mathrm{W}}\right\}$ is a myopic-farsighted stable set.

Proof. Since $\left\{\mu^{\mathrm{W}}\right\}$ is a singleton set, Condition (i) of Definition 2, internal stability, is satisfied. Condition (ii) of Definition 2, external stability, follows from Lemmas 2 and 4.

If the farsighted side of the market is desirable, then that side is able to induce its optimal stable matching, since $\left\{\mu^{\mathrm{W}}\right\}$ is a myopic-farsighted stable set. In order to obtain this result we have proved that, starting from any matching, we can always reach the woman-optimal stable matching by means of a myopic-farsighted improving path. The fact that we can reach a designated core element is striking, certainly when taking into account that the celebrated result of Roth and Vande Vate (1990) only shows that some core element can always be reached by means of a myopic improving path from any initial matching.

Several results in the matching literature, see for instance Diamantoudi and Xue (2003), Ehlers (2007), Mauleon, Vannetelbosch, and Vergote (2011), and Herings, Mauleon, and Vannetelbosch (2017) among others, point towards the core as the set of reasonable outcomes, but are not able to discriminate between different core elements. However, in these papers, no heterogeneity regarding the degree of farsightedness of players on the two sides of the market was taken into account and it was assumed that both sides were either myopic or farsighted. In case one side 
of the market is myopic whereas the other side contains some farsighted players, Example 2 illustrates that the optimal stable matching for the farsighted side may be the only myopic-farsighted stable set, whereas Theorem 3 provides a condition under which the most farsighted side can always reach its optimal stable matching.

\section{All Women Are Farsighted}

In Section 5 we have considered the case where all men are myopic, whereas any given woman can be either myopic or farsighted. In this section we assume all women to be farsighted, whereas any given man can be either myopic or farsighted. We prove first that the woman-optimal stable matching $\mu^{\mathrm{W}}$ can be reached from any matching $\mu$ different from $\mu^{\mathrm{W}}$ with the property that, for every $w \in W, \mu^{\mathrm{W}}(w) \succeq_{w} \mu(w)$. This covers the case where $\mu$ is a core element different from $\mu^{\mathrm{W}}$.

Lemma 5. Let $(M, W, \succ)$ be a marriage problem with set of farsighted players $F \supset$ $W$. For every $\mu \in \mathcal{M} \backslash\left\{\mu^{\mathrm{W}}\right\}$ such that, for every $w \in W, \mu^{\mathrm{W}}(w) \succeq_{w} \mu(w)$ it holds that $\mu^{\mathrm{W}} \in h(\mu)$.

Proof. Let $\mu \in \mathcal{M} \backslash\left\{\mu^{\mathrm{W}}\right\}$ be a matching such that, for every $w \in W, \mu^{\mathrm{W}}(w) \succeq_{w}$ $\mu(w)$. We construct a myopic-farsighted improving path $\mu_{0}, \ldots, \mu_{L}$ from $\mu_{0}=\mu$ to $\mu_{L}=\mu^{\mathrm{W}}$. Let

$$
W^{1}=\left\{w \in W \mid \mu^{\mathrm{W}}(w) \succ_{w} \mu(w) \text { and } \mu(w) \in M\right\}
$$

be the, possibly empty, set of women who strictly prefer $\mu^{\mathrm{W}}(w)$ to $\mu(w)$ and who are married at $\mu$. Let $k^{1}$ be the cardinality of $W^{1}$. Take an arbitrary order of the women in $W^{1}$, say $w_{0}, \ldots, w_{k^{1}-1}$.

For $\ell \in\left\{0, \ldots, k^{1}-1\right\}$, we define the matching $\mu_{\ell+1}=\mu_{\ell}-\left(\mu_{\ell}\left(w_{\ell}\right), w_{\ell}\right)$, so the $k^{1}$ women in $W^{1}$ sequentially destroy their matches. We show that the sequence of matchings $\mu_{0}, \ldots, \mu_{k^{1}}$ is the first part of a myopic-farsighted improving path from $\mu$ to $\mu^{\mathrm{W}}$ by showing that for every $\ell \in\left\{0, \ldots, k^{1}-1\right\}$ we have $\mu_{L}\left(w_{\ell}\right)=\mu^{\mathrm{W}}\left(w_{\ell}\right) \succ_{w_{\ell}}$ $\mu_{\ell}\left(w_{\ell}\right)$. This follows since $\mu_{\ell}\left(w_{\ell}\right)=\mu\left(w_{\ell}\right)$ and by the definition of $W^{1}$.

At $\mu_{k^{1}}$ every woman is either single or married to her partner at $\mu^{\mathrm{W}}$. It then follows that at $\mu_{k^{1}}$ every man is either single or married to his partner at $\mu^{\mathrm{W}}$. Let

$$
W^{2}=\left\{w \in W \mid \mu^{\mathrm{W}}(w) \succ_{w} \mu_{k^{1}}(w)\right\}
$$


be the set of women that strictly prefer the match at $\mu^{\mathrm{W}}$ to being single. Let $k^{2}$ be the cardinality of the set $W^{2}$. Take an arbitrary order of the women in $W^{2}$, say $w_{0}, \ldots, w_{k^{2}-1}$. For $\ell=0, \ldots, k^{2}-1$, we define $m_{\ell}=\mu^{\mathrm{W}}\left(w_{\ell}\right)$.

For $\ell=0, \ldots, k^{2}-1$, we define the matching $\mu_{k^{1}+\ell+1}=\mu_{k^{1}+\ell}+\left(m_{\ell}, w_{\ell}\right)$, so the $k^{2}$ women in $W^{2}$ sequentially marry their partner at $\mu^{\mathrm{W}}$. It holds that $\mu_{L}=$ $\mu_{k^{1}+k^{2}}=\mu^{\mathrm{W}}$. Observe that the sequence of matchings $\mu_{k^{1}}, \ldots, \mu_{k^{1}+k^{2}}$ is the final part of a myopic-farsighted improving path from $\mu$ to $\mu^{\mathrm{W}}$ since it holds that, for every $\ell \in\left\{0, \ldots, k^{2}-1\right\}$,

$$
\begin{aligned}
\mu_{k^{1}+\ell+1}\left(m_{\ell}\right) & =\mu^{\mathrm{W}}\left(m_{\ell}\right) \succ_{m_{\ell}} m_{\ell}=\mu_{k^{1}+\ell}\left(m_{\ell}\right), \quad \text { if } m_{\ell} \in M \backslash F, \\
\mu_{L}\left(m_{\ell}\right) & =\mu^{\mathrm{W}}\left(m_{\ell}\right) \succ_{m_{\ell}} m_{\ell}=\mu_{k^{1}+\ell}\left(m_{\ell}\right), \quad \text { if } m_{\ell} \in F, \\
\mu_{L}\left(w_{\ell}\right) & =\mu^{\mathrm{W}}\left(w_{\ell}\right) \succ_{w_{\ell}} w_{\ell}=\mu_{k^{1}+\ell}\left(w_{\ell}\right) .
\end{aligned}
$$

As in the proof of Lemma 2, the proof of Lemma 5 first identifies the set $W^{1}$ of women that strictly prefer $\mu^{\mathrm{W}}$ to $\mu$ and that are not single at $\mu$. Since all women in $W^{1}$ are farsighted, they are willing to divorce their men. The resulting matching is such that all women are either married to their partner at $\mu^{\mathrm{W}}$ or are single. The latter women now marry their partner at $\mu^{\mathrm{W}}$.

We now turn to the case where the matching $\mu$ is such that some women prefer $\mu$ to $\mu^{\mathrm{W}}$, so the set of women $W(\mu)$ that strictly prefer their match at $\mu$ to the one at $\mu^{\mathrm{W}}$ is not empty.

Lemma 6. Let $(M, W, \succ)$ be a marriage problem with set of farsighted players $F \supset$ $W$. For every $\mu \in \mathcal{M}$ such that $W(\mu) \neq \emptyset$ it holds that $\mu^{\mathrm{W}} \in h(\mu)$.

Proof. We construct a myopic-farsighted improving path $\mu_{0}, \ldots, \mu_{L}$ from $\mu_{0}=\mu$ to $\mu_{L}=\mu^{\mathrm{W}}$. Let

$$
W^{1}=\left\{w \in \mu(M) \mid \mu(w) \succ_{\mu(w)} w \text { or } w \succ_{w} \mu(w)\right\}
$$

be the, possibly empty, set of women that are involved in a match that is not individually rational for at least one of the partners and denote the cardinality of $W^{1}$ by $k^{1}$. Take an arbitrary order of the women in $W^{1}$, say $w_{0}, \ldots, w_{k^{1}-1}$. For $\ell \in\left\{0, \ldots, k^{1}-1\right\}$, we define $m_{\ell}=\mu\left(w_{\ell}\right)$ to be the man married to $w_{\ell}$ at $\mu$ and we define the matching $\mu_{\ell+1}=\mu_{\ell}-\left(m_{\ell}, w_{\ell}\right)$, so the player who is involved in a match under $\mu$ that is not individually rational destroys his or her link. We argue that the 
sequence of matchings $\mu_{0}, \ldots, \mu_{k^{1}}$ is the first part of a myopic-farsighted improving path from $\mu$ to $\mu^{\mathrm{W}}$.

Let some $\ell \in\left\{0, \ldots, k^{1}-1\right\}$ be given. It holds that $m_{\ell} \succ_{m_{\ell}} w_{\ell}$ or $w_{\ell} \succ_{w_{\ell}} m_{\ell}$. In the former case, we have that

$$
\begin{aligned}
\mu_{\ell+1}\left(m_{\ell}\right) & =m_{\ell} \succ_{m_{\ell}} \mu_{\ell}\left(m_{\ell}\right), & & \text { if } m_{\ell} \in M \backslash F, \\
\mu_{L}\left(w_{\ell}\right) & =\mu^{\mathrm{W}}\left(m_{\ell}\right) \succeq_{m_{\ell}} m_{\ell} \succ_{m_{\ell}} \mu_{\ell}\left(m_{\ell}\right), & & \text { if } m_{\ell} \in F,
\end{aligned}
$$

whereas in the latter case it holds that

$$
\mu_{L}\left(w_{\ell}\right)=\mu^{\mathrm{W}}\left(w_{\ell}\right) \succeq_{w_{\ell}} w_{\ell} \succ_{w_{\ell}} \mu_{\ell}\left(w_{\ell}\right)
$$

so the conditions of Definition 1 are satisfied.

Let

$$
W^{2}=\left\{w \in W\left(\mu_{k^{1}}\right) \mid \mu_{k^{1}}(w) \in F\right\}
$$

be the, possibly empty, set of women that prefer their match at $\mu_{k^{1}}$ to the one at $\mu^{\mathrm{W}}$ and that are matched to a farsighted man. We denote the cardinality of $W^{2}$ by $k^{2}$ and take an arbitrary order of the women in $W^{2}$, say $w_{0}, \ldots, w_{k^{2}-1}$. For $\ell \in\left\{0, \ldots, k^{2}-1\right\}$, we define $m_{\ell}=\mu_{k^{1}}\left(w_{\ell}\right)$ to be the man married to $w_{\ell}$ at $\mu_{k^{1}}$ and we define the matching $\mu_{k^{1}+\ell+1}=\mu_{k^{1}+\ell}-\left(m_{\ell}, w_{\ell}\right)$, so man $m_{\ell}$ destroys his match with $w_{\ell}$. We argue that the sequence of matchings $\mu_{k^{1}}, \ldots, \mu_{k^{1}+k^{2}}$ is the second part of a myopic-farsighted improving path from $\mu$ to $\mu^{\mathrm{W}}$.

Let some $\ell \in\left\{0, \ldots, k^{2}-1\right\}$ be given. Since $m_{\ell} \succ_{w_{\ell}} \mu^{\mathrm{W}}\left(w_{\ell}\right)$ and $\mu^{\mathrm{W}}$ is a core element, it holds that

$$
\mu_{L}\left(m_{\ell}\right)=\mu^{\mathrm{W}}\left(m_{\ell}\right) \succ_{m_{\ell}} w_{\ell}=\mu_{k^{1}+\ell}\left(m_{\ell}\right),
$$

so the conditions of Definition 1 are satisfied.

Consider the set $W\left(\mu_{k^{1}+k^{2}}\right)$ of women that strictly prefer $\mu_{k^{1}+k^{2}}(w)$ to $\mu^{\mathrm{W}}(w)$. By construction of $\mu_{k^{1}+k^{2}}$ it holds that the set $\mu_{k^{1}+k^{2}}\left(W\left(\mu_{k^{1}+k^{2}}\right)\right)$ of men married to a woman in $W\left(\mu_{k^{1}+k^{2}}\right)$ are all myopic. For $\ell \in\{0,1, \ldots\}$, whenever $W\left(\mu_{k^{1}+k^{2}+\ell}\right) \neq \emptyset$, select some

$$
\left(m_{\ell}, w_{\ell}\right) \in\left(\mu_{k^{1}+k^{2}+\ell}\left(W\left(\mu_{k^{1}+k^{2}+\ell}\right)\right)\right) \times\left(W \backslash W\left(\mu_{k^{1}+k^{2}+\ell}\right)\right)
$$

that blocks $\mu_{k^{1}+k^{2}+\ell}$. Such a pair $\left(m_{\ell}, w_{\ell}\right)$ is guaranteed to exist by Lemma 3 . We define the matching $\mu_{k^{1}+k^{2}+\ell+1}=\mu_{k^{1}+k^{2}+\ell}+\left(m_{\ell}, w_{\ell}\right)$. 
We argue next that after a finite number of steps, say $k^{3}, W\left(\mu_{k^{1}+k^{2}+k^{3}}\right)=\emptyset$. Since for every $\ell \geq 0$, the man involved in the block is married to a woman in $W\left(\mu_{k^{1}+k^{2}+\ell}\right)$, it follows that the cardinality of the set $\mu_{k^{1}+k^{2}+\ell}\left(W\left(\mu_{k^{1}+k^{2}+\ell}\right)\right)$ is weakly decreasing in $\ell$ and that these sets are nested in one another. The only possibility for the cardinality of this set to remain the same is that woman $w_{\ell}$ is single under $\mu_{k^{1}+k^{2}+\ell}$. In that case, it holds that $\mu_{k^{1}+k^{2}+\ell+1}\left(m_{\ell}\right)=w_{\ell} \succ_{m_{\ell}} \mu_{k^{1}+k^{2}+\ell}\left(m_{\ell}\right)$ and, for every $m \in \mu_{k^{1}+k^{2}+\ell}\left(W\left(\mu_{k^{1}+k^{2}+\ell}\right)\right) \backslash\left\{m_{\ell}\right\}, \mu_{k^{1}+k^{2}+\ell+1}(m)=\mu_{k^{1}+k^{2}+\ell}(m)$. Man $m_{\ell}$ is strictly improving and all other men in $\mu_{k^{1}+k^{2}+\ell}\left(W\left(\mu_{k^{1}+k^{2}+\ell}\right)\right)$ remain married to the same partner. It follows that cycling is impossible, so after a finite number of steps $k^{3}$ we have $W\left(\mu_{k^{1}+k^{2}+k^{3}}\right)=\emptyset$.

We argue that the sequence of matchings $\mu_{k^{1}+k^{2}}, \ldots, \mu_{k^{1}+k^{2}+k^{3}}$ is the third part of a myopic-farsighted improving path from $\mu$ to $\mu^{\mathrm{W}}$.

Let some $\ell \in\left\{0, \ldots, k^{3}-1\right\}$ be given. Since the set of men $\mu_{k^{1}+k^{2}+\ell}\left(W\left(\mu_{k^{1}+k^{2}+\ell}\right)\right)$ is a subset of $\mu_{k^{1}+k^{2}}\left(W\left(\mu_{k^{1}+k^{2}}\right)\right)$, it holds that $m_{\ell}$ is myopic. Since $\left(m_{\ell}, w_{\ell}\right)$ blocks $\mu_{k^{1}+k^{2}+\ell}$, it follows that

$$
\mu_{k^{1}+k^{2}+\ell+1}\left(m_{\ell}\right) \succ_{m_{\ell}} \mu_{k^{1}+k^{2}+\ell}\left(m_{\ell}\right) .
$$

It holds that $w_{\ell} \in W \backslash W\left(\mu_{k^{1}+k^{2}+\ell}\right)$, so

$$
\mu_{L}\left(w_{\ell}\right)=\mu^{\mathrm{W}}\left(w_{\ell}\right) \succeq_{w_{\ell}} \mu_{\ell}\left(w_{\ell}\right)
$$

The conditions of Definition 1 are therefore satisfied.

Since $W\left(\mu_{k^{1}+k^{2}+k^{3}}\right)=\emptyset$, the matching $\mu_{k^{1}+k^{2}+k^{3}}$ is either equal to $\mu^{\mathrm{W}}$ or satisfies the assumptions of Lemma 5 . In the former case we are done, in the latter case we proceed as in the proof of Lemma 5 to complete the construction of the myopicfarsighted improving path leading to $\mu^{\mathrm{W}}$.

The proof of Lemma 6 proceeds as follows. As in the proof of Lemma 4, in order to reach $\mu^{\mathrm{W}}$ departing from $\mu$, first the matches that are not individually rational at $\mu$ are destroyed until we arrive at an individually rational matching $\mu_{k^{1}}$. We then identify all the women that prefer $\mu_{k^{1}}$ to $\mu^{\mathrm{W}}$ and are married to a farsighted man. Since $\mu^{\mathrm{W}}$ is a core element, it follows that such a farsighted man prefers $\mu^{\mathrm{W}}$ to $\mu_{k^{1}}$ and is willing to destroy the match with his partner. In this way we obtain a matching $\mu_{k^{1}+k^{2}}$ such that all women that prefer $\mu_{k^{1}+k^{2}}$ to $\mu^{\mathrm{W}}$ are married to a myopic man. From here, we proceed essentially in the same way as in Lemma 4. 
From Lemmas 5 and 6 , it follows easily that $\left\{\mu^{\mathrm{W}}\right\}$ is a myopic-farsighted stable set.

Theorem 4. Let $(M, W, \succ)$ be a marriage problem with set of farsighted players $F \supset W$. Then $\left\{\mu^{\mathrm{W}}\right\}$ is a myopic-farsighted stable set.

Proof. Since $\left\{\mu^{\mathrm{W}}\right\}$ is a singleton set, Condition (i) of Definition 2, internal stability, is satisfied. Condition (ii) of Definition 2, external stability, follows from Lemmas 5 and 6.

We have shown that in case all women are farsighted, they can achieve the woman-optimal stable matching, irrespective of the farsightedness of the men.

\section{Discussion}

In this section we discuss the robustness of our main result by answering some open questions. Is $\left\{\mu^{\mathrm{W}}\right\}$ the only myopic-farsighted stable set under the conditions of Theorems 3 and 4? The answer to this question is negative, as illustrated by Example 4. In Example 4, we assume that the farsighted players coincide with the women, $F=W$, thereby satisfying the conditions of both Theorems 3 and 4 . This example illustrates that $\left\{\mu^{\mathrm{M}}\right\}$ can be a myopic-farsighted stable set as well, which implies that it is possible to reach the man-optimal stable matching $\mu^{\mathrm{M}}$ from $\mu^{\mathrm{W}}$.

Example 4. Consider the marriage problem $(M, W, \succ)$, where $M=\left\{m_{1}, m_{2}, m_{3}\right\}$ and $W=\left\{w_{1}, w_{2}, w_{3}\right\}$ and the preferences of the players are as follows:

\begin{tabular}{|c|c|c|c|c|}
\hline$m_{1}$ & $m_{2}$ & $m_{3}$ & $w_{1}$ & $\underline{w_{2}}$ \\
\hline$w_{1}$ & $w_{3}$ & $w_{1}$ & $m_{1}$ & $m_{2}$ \\
\hline$w_{2}$ & $w_{1}$ & $w_{2}$ & $m_{2}$ & $m_{3}$ \\
\hline$w_{3}$ & $w_{2}$ & $w_{3}$. & $m_{3}$ & $m_{1}$ \\
\hline
\end{tabular}

We assume $F=W$, so all women are farsighted and all men are myopic. It is easily verified that this example satisfies the conditions of both Theorems 3 and 4. Using the deferred acceptance algorithm of Gale and Shapley (1962) with men proposing and with women proposing, it is immediate that the man-optimal and woman-optimal stable matchings are given by

$$
\begin{array}{ll}
\mu^{\mathrm{M}}\left(m_{1}\right)=w_{1}, & \mu^{\mathrm{W}}\left(m_{1}\right)=w_{1}, \\
\mu^{\mathrm{M}}\left(m_{2}\right)=w_{3}, & \mu^{\mathrm{W}}\left(m_{2}\right)=w_{2}, \\
\mu^{\mathrm{M}}\left(m_{3}\right)=w_{2} . & \mu^{\mathrm{W}}\left(m_{3}\right)=w_{3},
\end{array}
$$


We first argue that $\mu^{\mathrm{M}} \in h\left(\mu^{\mathrm{W}}\right)$. To verify this assertion, consider the myopicfarsighted improving path $\mu_{0}, \ldots, \mu_{4}$ with $\mu_{0}=\mu^{\mathrm{W}}$ and $\mu_{4}=\mu^{\mathrm{M}}$, where $\mu_{1}=$ $\mu_{0}+\left(m_{2}, w_{1}\right), \mu_{2}=\mu_{1}+\left(m_{3}, w_{2}\right), \mu_{3}=\mu_{2}+\left(m_{2}, w_{3}\right)$, and $\mu_{4}=\mu_{3}+\left(m_{1}, w_{1}\right)$. For an illustration, see Figure 4.

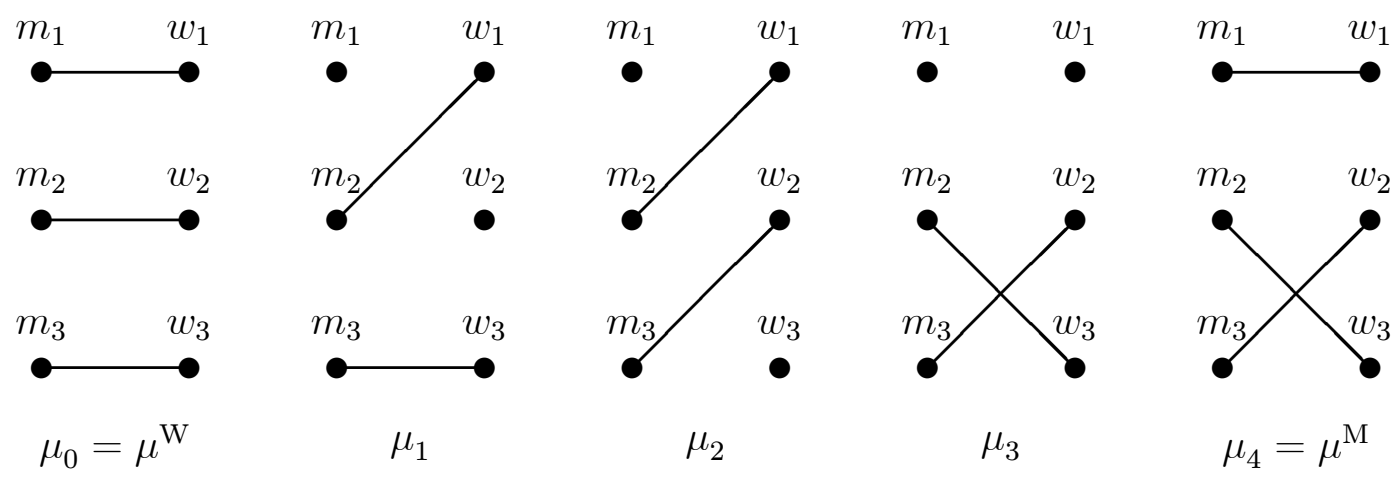

Figure 4: Myopic-farsighted improving path in Example 4 to move from $\mu^{\mathrm{W}}$ to $\mu^{\mathrm{M}}$.

Since $m_{2}$ strictly prefers $\mu_{1}\left(m_{2}\right)=w_{1}$ to $\mu_{0}\left(m_{2}\right)=w_{2}$ and the farsighted woman $w_{1}$ is indifferent between $\mu_{4}$ and $\mu_{0}$, the addition of link $\left(m_{2}, w_{1}\right)$ to $\mu_{0}$ satisfies Condition (ii) of Definition 1. Since now at $\mu_{1}$ woman $w_{2}$ has become unmatched, she is willing to form a link with $m_{3}$, her partner in the end matching of the sequence, moving from $\mu_{1}$ to $\mu_{2}$. Since $\mu_{2}\left(m_{3}\right)=w_{2} \succ_{m_{2}} w_{3}=\mu_{1}\left(m_{3}\right)$, this is also a myopic improvement for $m_{3}$. Since now at $\mu_{2}$ women $w_{3}$ is unmatched, she is willing to team up with $m_{2}$, her partner in the end matching of the sequence, leading to the matching $\mu_{3}$. Since $\mu_{3}\left(m_{2}\right)=w_{3} \succ_{m_{2}} w_{1}=\mu_{2}\left(m_{2}\right)$, this is also a myopic improvement for $m_{2}$. Man $m_{1}$ and woman $w_{1}$ are both single at $\mu_{3}$ and are both happy to marry, which moves them to the end matching $\mu_{4}=\mu^{\mathrm{M}}$.

Consider next any matching $\mu \in \mathcal{M} \backslash\left\{\mu^{\mathrm{M}}, \mu^{\mathrm{W}}\right\}$. We argue that $\mu^{\mathrm{M}} \in h(\mu)$ by constructing a myopic-farsighted improving path $\mu_{0}, \ldots, \mu_{L}$ with $\mu_{0}=\mu$ and $\mu_{L}=\mu^{\mathrm{M}}$.

Assume first that $\mu\left(m_{1}\right) \neq w_{1}$. We define $\mu_{1}=\mu_{0}+\left(m_{1}, w_{1}\right)$. Since $w_{1}$ is the best partner for $m_{1}$, this is clearly a myopic improvement for $m_{1}$. Since $m_{1}$ is the best partner for $w_{1}$ and $\mu_{L}\left(w_{1}\right)=\mu^{\mathrm{M}}\left(w_{1}\right)=m_{1}$, this is a farsighted improvement for $w_{1}$.

\footnotetext{
If $\mu_{1}=\mu^{\mathrm{M}}$, then we have shown that $\mu^{\mathrm{M}} \in h(\mu)$.

If $\mu_{1}=\mu^{\mathrm{W}}$, then following the myopic-farsighted improving path from $\mu^{\mathrm{W}}$ to $\mu^{\mathrm{M}}$
} 
constructed at the beginning of the example, we also have $\mu^{\mathrm{M}} \in h(\mu)$.

If $\mu_{1} \in \mathcal{M} \backslash\left\{\mu^{\mathrm{M}}, \mu^{\mathrm{W}}\right\}$, then $w_{2}$ or $w_{3}$ is single under $\mu_{1}$. If $w_{2}$ is single, then let her marry $m_{3}$ and move to $\mu_{2}=\mu_{1}+\left(m_{3}, w_{2}\right)$. Since at $\mu_{1}$ man $m_{3}$ is not married to $w_{1}$, this is a myopic improvement for $m_{3}$. It is also clearly a farsighted improvement for $w_{2}$. If $w_{2}$ is not single, but $w_{3}$ is, then let her marry $m_{2}$ and move to $\mu_{2}=\mu_{1}+\left(m_{2}, w_{3}\right)$. Since $w_{3}$ is the preferred partner of $m_{2}$, this is clearly a myopic improvement for $m_{2}$. It is also clearly a farsighted improvement for $w_{3}$. Either $\mu_{2}=\mu^{\mathrm{M}}$ and we are done, or $\mu^{2}$ consists of two matched pairs $\left(m_{1}, w_{1}\right)$ and $\left(m_{3}, w_{2}\right)$, both being part of $\mu^{\mathrm{M}}$, and two single players, $m_{2}$ and $w_{3}$. In this case, we form the missing pair $\left(m_{2}, w_{3}\right)$ from $\mu^{\mathrm{M}}$ and move from $\mu_{2}$ to $\mu_{3}=\mu^{\mathrm{M}}$. This completes the construction of the myopic-farsighted improving path to $\mu^{\mathrm{M}}$ for the case $\mu\left(m_{1}\right) \neq w_{1}$.

Assume next that $\mu\left(m_{1}\right)=w_{1}$. We can then proceed with the myopic-farsighted improving path starting from $\mu_{1}$ as constructed in the previous paragraph, with $\mu_{1}$ being replaced by $\mu$.

The singleton set $V=\left\{\mu^{\mathrm{M}}\right\}$ trivially satisfies Condition (i), internal stability, of Definition 2. Since we have shown that $\mu^{\mathrm{M}} \in h(\mu)$ for every $\mu \neq \mu^{\mathrm{M}}$, it also satisfies Condition (ii) of Definition 2, external stability. It follows that $V=\left\{\mu^{\mathrm{M}}\right\}$ is a myopic-farsighted stable set.

Example 4 shows that even though $\left\{\mu^{\mathrm{W}}\right\}$ is the focal myopic-farsighted stable set, in some examples there are other myopic-farsighted stable sets as well.

Example 5 has the same primitives as Example 4 and demonstrates that in some cases even a non-core element can serve as a myopic-farsighted stable set.

Example 5. Let $(M, W, \succ)$ with $F=W$ be the marriage problem of Example 4 .

Consider now the matching $\mu^{\prime}$ illustrated in Figure 5 with

$$
\begin{aligned}
& \mu^{\prime}\left(m_{1}\right)=w_{1}, \\
& \mu^{\prime}\left(m_{2}\right)=m_{2}, \\
& \mu^{\prime}\left(m_{3}\right)=w_{3},
\end{aligned}
$$

that contains only two of the matches of the woman-optimal stable matching $\mu^{\mathrm{W}}$. The matching $\mu^{\prime}$ is not stable as $\left(m_{2}, w_{2}\right)$ blocks $\mu^{\prime}$.

We show next that $V=\left\{\mu^{\prime}\right\}$ satisfies external stability and is therefore a singleton myopic-farsighted stable set. 


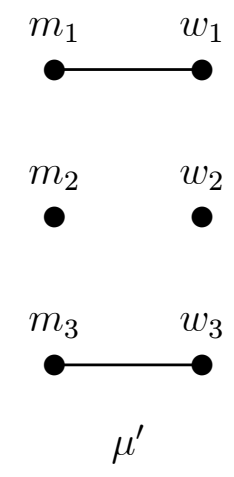

Figure 5: The matching $\mu^{\prime}$ of Example 5.

Take any $\mu \neq \mu^{\prime}$ and take $\mu_{0}=\mu$. We construct a myopic-farsighted improving path $\mu_{0}, \ldots \mu_{L}$ with $\mu_{L}=\mu^{\prime}$. If $w_{2}$ is married under $\mu$, then add the match $\left(\mu\left(w_{2}\right), w_{1}\right)$ to $\mu_{0}$ and move to $\mu_{1}=\mu_{0}+\left(\mu\left(w_{2}\right), w_{1}\right)$. Notice that marrying $w_{1}$ is a myopic improvement for $\mu\left(w_{2}\right)$ since $w_{1}$ is strictly preferred to $w_{2}$ by any man. Since $m_{1}$ is the best possible partner for woman $w_{1}$, she weakly prefers the end matching $\mu^{\prime}$ with $\mu^{\prime}\left(w_{1}\right)=m_{1}$ to $\mu_{0}\left(w_{1}\right)$ and is therefore willing to collaborate.

It holds that $w_{2}$ is not married under $\mu$ or has become single after the marriage of $\mu\left(w_{2}\right)$ and $w_{1}$. We therefore obtain a matching $\mu_{\ell}$ such that $\mu_{\ell}\left(w_{2}\right)=w_{2}$, where $\ell$ is either equal to 0 or 1 . If $\mu_{\ell}\left(w_{1}\right) \neq m_{1}$, then move to $\mu_{\ell+1}=\mu_{\ell}+\left(m_{1}, w_{1}\right)$. Since $w_{1}$ is the best possible partner for $m_{1}$, this is a myopic improvement for $m_{1}$. In case $\mu_{\ell}\left(w_{1}\right) \neq m_{1}$ it holds that $\mu^{\prime}\left(w_{1}\right)=m_{1} \succ_{w_{1}} \mu_{\ell}\left(w_{1}\right)$, so the marriage with $m_{1}$ is a farsighted improvement for $w_{1}$.

We now have a matching such that $m_{1}$ is married to $w_{1}$ and $w_{2}$ is single. If $w_{3}$ is married to $m_{3}$ then our matching is equal to $\mu^{\prime}$ and we are done. Otherwise, $m_{3}$ and $w_{3}$ are both single and they marry to arrive at $\mu^{\prime}$.

Notice that the matching $\mu^{\prime}$ contains a proper subset of the matches that are present in $\mu^{\mathrm{W}}$ and matches the farsighted woman with the same partner as in $\mu^{\mathrm{W}}$. The fact that a non-core element can yield a myopic-farsighted stable set is surprising. It follows from Herings, Mauleon, and Vannetelbosch (2017) that in case all players are myopic, the myopic-farsighted stable set coincides with the core. It follows from Mauleon, Vannetelbosch, and Vergote (2011) that in case all players are farsighted, the myopic-farsighted stable sets are the singleton sets containing a core-element. In case not all players have the same degree of farsightedness, an 
element outside the core may result.

\section{Conclusion}

Motivated by empirical and experimental evidence that agents have heterogeneous degrees of farsightedness, we study von Neumann Morgenstern stable sets for marriage problems in the presence of both myopic and farsighted players. To do so, we introduce the new notion of a myopic-farsighted improving path. A myopic-farsighted improving path is a sequence of matchings that can emerge when farsighted players form or destroy links based on the improvement the end matching offers relative to the current matching while myopic players form or destroy matches based on the improvement the resulting matching offers relative to the current matching.

The myopic-farsighted stable set corresponds to the von Neumann Morgenstern stable set based on myopic-farsighted improving paths. A myopic-farsighted stable set is therefore defined as a set of matchings such that there is no myopic-farsighted improving path from any matching in the set to another matching in the set (internal stability) and there is a myopic-farsighted improving path from any matching outside the set to some matching in the set (external stability).

The myopic-farsighted stable set bridges the case where all players are myopic and the case where all players are farsighted. It reduces to the pairwise CP vNM set of Herings, Mauleon, and Vannetelbosch (2017) when all players are myopic. Under these circumstances, the myopic-farsighted stable set is unique and is equal to the core. In case all players are farsighted, it corresponds to the vNM farsightedly stable set of Mauleon, Vannetelbosch, and Vergote (2011) under the formulation that only single players and pairs of players can create and destroy links. The myopicfarsighted stable sets with only farsighted players are characterized as the singletons containing a core element.

We then assume all men to be myopic, whereas any woman may be either farsighted or myopic. We provide a condition under which the woman-optimal stable matching is always a myopic-farsighted stable set. Hence, the most farsighted side of the market is favored in the sense that the presence of some farsighted women is enough to guarantee that the woman-optimal stable matching can always be reached, starting from any other matching, by means of a myopic-farsighted improving path.

We use the simplest example of a marriage problem with the preferences of men 
and women diametrically opposed to each other to show that the woman-optimal stable matching is the unique myopic-farsighted stable set, even when the core is not a singleton. We thereby provide a theory of 'equilibrium selection' for stable matchings that links the theoretical results regarding the stability of some selected stable matching with the experimental and empirical evidence that has analyzed whether markets systematically favor a selected stable matching with particular characteristics. However, other myopic-farsighted stable sets can exist consisting of a core element different from the woman-optimal matching or even of a non-core element. Thus, farsighted players cannot always guarantee to themselves a better stable matching than myopic players.

\section{Acknowledgments}

Vincent Vannetelbosch and Ana Mauleon are Senior Research Associates of the National Fund for Scientific Research (FNRS). Financial support from the Spanish Ministry of Economy and Competition under the project ECO2015-64467-R, from the Fonds de la Recherche Scientifique - FNRS under the grant J.0073.15 and from the Belgian French speaking community ARC project $n^{\circ} 15 / 20-072$ of Saint-Louis University - Brussels is gratefully acknowledged.

\section{References}

Basteck, C. and M. Mantovani (2016), "Cognitive Ability and Games of School Choice", DEMS Working Paper $N^{\circ} 343$, University of Milan-Bicocca.

Boudreau, J.W. (2011), "A Note on the Efficiency and Fairness of Decentralized Matching", Operations Research Letters 39, 231-233.

Chen, Y. and T. Sönmez (2006), "School Choice: An Experimental Study", Journal of Economic Theory 127, 202-231.

Chwe, M. S.-Y. (1994), "Farsighted Coalitional Stability", Journal of Economic Theory 63, 299-325.

Deemen, A.M.A. van (1991), "A Note on Generalized Stable Sets", Social Choice and Welfare 8, 255-260.

Diamantoudi, E., and L. Xue (2003), "Farsighted Stability in Hedonic Games", Social Choice and Welfare 21, 39-61. 
Echenique, F., A.J. Wilson and L. Yariv (2016), "Clearinghouses for Two-Sided Matching: An Experimental Study", Quantitative Economics 7, 449-482.

Echenique, F. and L. Yariv (2012), "An Experimental Study of Decentralized Matching", Working Paper, Caltech.

Ehlers, L. (2007), "Von Neumann-Morgenstern Stable Sets in Matching Problems", Journal of Economic Theory 134, 537-547.

Featherstone, C.R. and E. Mayefsky (2011), "Why Do Some Clearinghouses Yield Stable Outcomes? Experimental Evidence on Out-of-Equilibrium TruthTelling", Working Paper, Stanford University.

Featherstone, C.R. and M. Niederle (2011), "School Choice Mechanisms under Incomplete Information: An Experimental Investigation", Working Paper, Harvard Business School.

Gale, D., and L.S. Shapley (1962), "College Admissions and the Stability of Marriage", American Mathematical Monthly 69, 9-15.

Harrison, G. and K.A. McCabe (1992), "Testing Noncooperative Bargaining Theory in Experiments", Research in Experimental Economics 5, 137-169.

Harsanyi, J.C. (1974), "An Equilibrium-Point Interpretation of Stable Sets and a Proposed Alternative Definition", Management Science 20, 1472-1495.

Haruvy, E. and M.U. Ünver (2007) "Equilibrium Selection and the Role of Information in Repeated Matching Markets", Economics Letters 94, 284-289.

Herings, P.J.J., A. Mauleon and V. Vannetelbosch (2004), "Rationalizability for Social Environments", Games and Economic Behavior 49, 135-156.

Herings, P.J.J., A. Mauleon and V. Vannetelbosch (2009), "Farsightedly Stable Networks", Games and Economic Behavior 67, 526-541.

Herings, P.J.J., A. Mauleon and V. Vannetelbosch (2017), "Stable Sets in Matching Problems with Coalitional Sovereignty and Path Dominance", forthcoming in Journal of Mathematical Economics.

Hitsch, G.J., A. Hortaçsu and D. Ariely (2010), "Matching and Sorting in Online Dating", American Economic Review 100, 130-163. 
Kagel, J.H. and A.E. Roth (2000), "The Dynamics of Reorganization in Matching Markets: A Laboratory Experiment Motivated by a Natural Experiment", Quarterly Journal of Economics 115, 201-235.

Knuth, D.E. (1976), Marriages Stables, Les Presses de l'Université de Montreal, Montreal.

Mauleon, A., and V. Vannetelbosch (2004), "Farsightedness and Cautiousness in Coalition Formation Games with Positive Spillovers", Theory and Decision $56,291-324$.

Mauleon, A., V. Vannetelbosch and W. Vergote (2011), "Von Neumann-Morgenstern Farsightedly Stable Sets in Two-sided Matching", Theoretical Economics 6, $499-521$.

Nalbantian, H.R. and A. Schotter (1995), "Matching and Efficiency in the Baseball Free-Agent System: An Experimental Examination", Journal of Labor Economics 13, 1-31.

Page, F.H., Jr. and M. Wooders (2009), "Strategic Basins of Attraction, the Path Dominance Core, and Network Formation Games", Games and Economic Behavior 66, 462-487.

Page, F.H., Jr., M. Wooders, and S. Kamat (2005), "Networks and Farsighted Stability", Journal of Economic Theory 120, 257-269.

Pais, J. and Á. Pintér (2008), "School Choice and Information: An Experimental Study on Matching Mechanisms", Games and Economic Behavior 64, 303328.

Ray, D. and R. Vohra (2015), "The Farsighted Stable Set", Econometrica 83, 977-1011.

Roth, A.E. and M.A.O. Sotomayor (1990), Two-Sided Matching, A Study in Game-Theoretic Modeling and Analysis, Econometric Society Monographs No. 18, Cambridge University Press, Cambridge.

Roth, A.E. and J.H. Vande Vate (1990), "Random Paths to Stability in Twosided Matching", Econometrica 58, 1475-1480. 
Wako, J. (2010), "A Polynomial-Time Algorithm to Find von Neumann-Morgenstern Stable Matchings in Marriage Games", Algorithmica 58, 188-220.

Xue, L. (1998), "Coalitional Stability under Perfect Foresight", Economic Theory $11,603-627$. 